\title{
Trace radioactive impurities in final construction materials for EXO-200
}

D.S. Leonard ${ }^{\mathrm{a}}$, D. Auty ${ }^{\mathrm{b}, 1}$, T. Didberidze $^{\mathrm{b}}$, R. Gornea $^{\mathrm{c}, \mathrm{d}}$, P. Grinberg ${ }^{\text {e }}$, R. MacLellan ${ }^{\mathrm{f}}$, B. Methven ${ }^{\mathrm{e}}$, A. Piepke ${ }^{\mathrm{b}}$, J.-L. Vuilleumier ${ }^{\mathrm{g}}$, J.B. Albert ${ }^{\mathrm{h}}$, G. Anton ${ }^{\mathrm{i}}$, I. Badhrees ${ }^{\mathrm{c}}$, P.S. Barbeau ${ }^{\mathrm{j}}$, R. Bayerlein ${ }^{\mathrm{i}}$, D. Beck ${ }^{\mathrm{k}}$, V. Belov ${ }^{\ell}$, M. Breidenbach ${ }^{\mathrm{m}}$, T. Brunner ${ }^{\mathrm{n}, \mathrm{d}}$, G.F. $\mathrm{Cao}^{\circ}$, W.R. Cen ${ }^{\mathrm{o}}$, C. Chambers ${ }^{\mathrm{p}}$, B. Cleveland ${ }^{\mathrm{q}, \mathrm{r}}$, M. Coon $^{\mathrm{k}}$, A. Craycraft ${ }^{\mathrm{p}}$, W. Cree $^{c}$, T. Daniels ${ }^{\mathrm{m}}$, M. Danilov ${ }^{\ell, 2}$, S.J. Daugherty ${ }^{\mathrm{h}}$, J. Daughhetee $^{\mathrm{f}}$, J. Davis ${ }^{\mathrm{m}}$, S. Delaquis ${ }^{\mathrm{m}}$,

A. Der Mesrobian-Kabakian ${ }^{\mathrm{q}}$, R. DeVoe ${ }^{\mathrm{s}}$, J. Dilling ${ }^{\mathrm{d}}$, A. Dolgolenko ${ }^{\ell}$, M.J. Dolinski ${ }^{\mathrm{t}}$, W. Fairbank Jr. ${ }^{\mathrm{p}}$, J. Farine ${ }^{\mathrm{q}}$, S. Feyzbakhsh ${ }^{\mathrm{u}}$, P. Fierlinger ${ }^{\mathrm{v}}$, D. Fudenberg ${ }^{\mathrm{s}}$, K. Graham ${ }^{\mathrm{c}}$, G. Gratta ${ }^{\mathrm{s}}$, C. Hall ${ }^{\mathrm{w}}$, S. Herrin ${ }^{\mathrm{m}, 3}$, J. Hoessl ${ }^{\mathrm{i}}$, P. Hufschmidt ${ }^{\mathrm{i}}$,

M. Hughes ${ }^{\mathrm{b}}$, A. Jamil ${ }^{\mathrm{i}, \mathrm{s}}$, M.J. Jewell ${ }^{\mathrm{s}}$, A. Johnson ${ }^{\mathrm{m}}$, S. Johntson ${ }^{\mathrm{u}, 4}$, A. Karelin ${ }^{\ell}$, L.J. Kaufman ${ }^{\text {h}}$, T. Koffas ${ }^{\mathrm{c}}$, S. Kravitz ${ }^{\text {, }}$ R. Krücken ${ }^{\mathrm{d}}$, A. Kuchenkov ${ }^{\ell}$, K.S. Kumar ${ }^{\mathrm{x}}$,

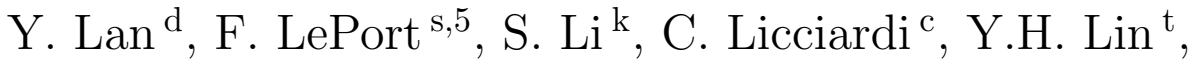
D. Mackay ${ }^{\mathrm{m}, 6}$, M.G. Marino ${ }^{\mathrm{v}}$, T. Michel $^{\mathrm{i}}$, B. Mong ${ }^{\mathrm{m}}$,

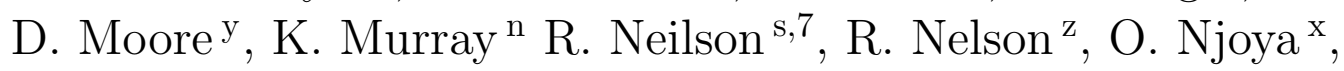
A. Odian ${ }^{\mathrm{m}}$, I. Ostrovskiy ${ }^{\mathrm{b}}$, A. Pocar ${ }^{\mathrm{u}}$, K. Pushkin ${ }^{\mathrm{b}, 8}$, F. Retière ${ }^{\mathrm{d}}$, P.C. Rowson ${ }^{\mathrm{m}}$, J.J. Russell ${ }^{\mathrm{m}}$, A. Schubert ${ }^{\mathrm{s}}$, D. Sinclair ${ }^{\mathrm{c}, \mathrm{d}}$, E. Smith ${ }^{\mathrm{t}, 9}$, V. Stekhanov ${ }^{\ell}$, M. Tarka ${ }^{\mathrm{x}}$, T. Tolba ${ }^{\circ}$, R. Tsang ${ }^{\mathrm{b}, 10}$, M. Wagenpfeil ${ }^{\mathrm{i}}$, A. Waite $^{\mathrm{m}}$, J. Walton $^{\mathrm{k}}{ }^{\text {, T. Walton }}{ }^{\mathrm{p}}, \mathrm{K}$. Wamba ${ }^{\mathrm{m}, 11}$, , M. Weber $^{\mathrm{s}}$, L.J. Wen ${ }^{\circ}$, U. Wichoski ${ }^{\mathrm{q}}$, J.Wodin ${ }^{\mathrm{m}, 12}$,L. Yang ${ }^{\mathrm{k}}$, Y.-R. Yen ${ }^{\mathrm{t}}$, O.Ya. Zeldovich ${ }^{\ell}$, J. Zettlemoyer ${ }^{\mathrm{h}}$, T. Ziegler ${ }^{\mathrm{i}}$

${ }^{a} I B S$ Center for Underground Physics, Daejeon 34047, Korea

${ }^{\mathrm{b}}$ Department of Physics and Astronomy, University of Alabama, Tuscaloosa, Alabama 35487, USA

' Physics Department, Carleton University, Ottawa, Ontario K1S 5B6, Canada

d TRIUMF, Vancouver, British Columbia V6T 2A3, Canada 
${ }^{\mathrm{e}}$ Measurement Science and Standards, National Research Council Canada, Ottawa ON, Canada

${ }^{\mathrm{f}}$ Physics Department, University of South Dakota, Vermillion, South Dakota 57069, USA

${ }^{g}$ LHEP, Albert Einstein Center, University of Bern, Bern, Switzerland

${ }^{\mathrm{h}}$ Physics Department and CEEM, Indiana University, Bloomington, Indiana 47405, USA

${ }^{\mathrm{i}}$ Erlangen Centre for Astroparticle Physics (ECAP),

Friedrich-Alexander-University Erlangen-Nürnberg, Erlangen 91058, Germany

${ }^{\mathrm{j}}$ Department of Physics, Duke University, and Triangle Universities Nuclear

Laboratory (TUNL), Durham, North Carolina 27708, USA

${ }^{\mathrm{k}}$ Physics Department, University of Illinois, Urbana-Champaign, Illinois 61801, $U S A$

${ }^{\ell}$ Institute for Theoretical and Experimental Physics, Moscow, Russia

${ }^{\mathrm{m}}$ SLAC National Accelerator Laboratory, Menlo Park, California 94025, USA

${ }^{n}$ Physics Department, McGill University, Montréal, Québec H3A 2T8, Canada

${ }^{\circ}$ Institute of High Energy Physics, Beijing, China

p Physics Department, Colorado State University, Fort Collins, Colorado 80523, $U S A$

${ }^{\mathrm{q}}$ Department of Physics, Laurentian University, Sudbury, Ontario P3E 2C6, Canada

${ }^{\mathrm{r}}$ SNOLAB, Sudbury, Ontario P3Y 1N2, Canada

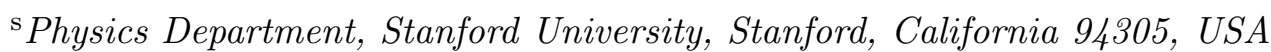

${ }^{\mathrm{t}}$ Department of Physics, Drexel University, Philadelphia, Pennsylvania 19104, $U S A$

u Amherst Center for Fundamental Interactions and Physics Department, University of Massachusetts, Amherst, MA 01003, USA

v Technische Universität München, Physikdepartment and Excellence Cluster Universe, Garching 80805, Germany

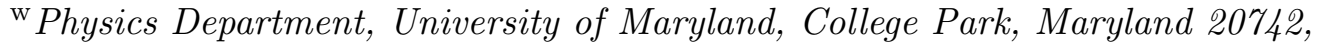
$U S A$

${ }^{\mathrm{x}}$ Department of Physics and Astronomy, Stony Brook University, SUNY, Stony Brook, New York 11794, USA

y Department of Physics, Yale University, New Haven, Connecticut 06511, USA

${ }^{\mathrm{z}}$ Waste Isolation Pilot Plant, Carlsbad, New Mexico 88220, USA

\section{Abstract}

We report results from a systematic measurement campaign conducted to identify 
low radioactivity materials for the construction of the EXO-200 double beta decay experiment. Partial results from this campaign have already been reported in a 2008 paper by the EXO collaboration. Here we release the remaining data, collected since 2007, to the public. The data reported were obtained using a variety of analytic techniques. The measurement sensitivities are among the best in the field. Construction of the EXO-200 detector has been concluded, and Phase-I data was taken from 2011 to 2014 . The detector's extremely low background implicitly verifies the measurements and the analysis assumptions made during construction and reported in this paper.

Key words: radiopurity, ultra-trace analysis, neutron activation analysis, mass spectrometry, mass spectroscopy, germanium counting, alpha counting, low background, double beta decay, EXO, EXO-200

PACS: 82.80.Jp, 14.60.Pq, 23.40.-s, 23.40.Bw

\section{Introduction}

Low energy, low-rate counting experiments such as searches for double beta decay, dark matter, and neutrino oscillations rely on access to construction materials containing the smallest possible amounts of radioactivity. The presence of radioactivity near the detector, even in ultra-trace concentrations, often causes unwanted background, potentially limiting the scientific reach of these experiments. The access to a range of low activity materials is, therefore, enabling science.

Specifically, this work was motivated by the Enriched Xenon Observatory (EXO), a multi-stage experimental research program with the purpose of detecting rare double beta decays of ${ }^{136} \mathrm{Xe}$ [1]. With EXO-200, we search for these decays in an underground cryogenic time-projection chamber (TPC) filled

1 Now at University of Alberta, Edmonton, Canada

2 Now at P. N. Lebedev Physical Institute of the Russian Academy of Sciences, Moscow, Russia

3 Now at 23andMe, Inc, Mountain View, CA, USA

4 Now at Argonne National Laboratory, Argonne, Illinois USA

5 Now an independent consultant

6 Now at KLA-Tencor, Milpitas, CA, USA

7 Now at Drexel University, Philadelphia, Pennsylvania, USA

8 Now at University of Michigan, Ann Arbor, Michigan, USA

9 Now at Indiana University, Bloomington, IN, USA

${ }^{10}$ Now at Pacific Northwest National Laboratory, Richland, Washington, USA

${ }^{11}$ Now at Gavilan College, Gilroy, CA, USA

${ }^{12}$ Now at SRI International, Menlo Park, CA, USA 
with approximately $110 \mathrm{~kg}$ of active liquid xenon enriched to $80 \%$ in ${ }^{136} \mathrm{Xe}$. In Ref. [2] we reported on a campaign of measurements of radioactive impurities in potential construction materials for the purpose of achieving the low background rates required for successful operation. Similar measurement campaigns have been published for rare-event search efforts $[3,4,5,6,7,8,9,10]$. Here we augment the previously reported measurements with results obtained during the final stages of design and construction of EXO-200. Measurement techniques and conditions were generally the same as those described in Ref. [2]. As in the previous work, the radio-assay campaign described here focuses on natural radioactivity, namely ${ }^{40} \mathrm{~K},{ }^{232} \mathrm{Th}$ and ${ }^{238} \mathrm{U}$.

EXO-200 started taking data in 2011. The experiment has been described in detail in [11]. The experiment performed the first observation of the twoneutrino double beta decay of ${ }^{136} \mathrm{Xe}[12$, placed stringent limits on the neutrinoless decay mode [13,14, and reported the most precise determination of any two-neutrino double beta decay rate [15]. The background event rate of $R_{b}=(1.7 \pm 0.2) \cdot 10^{-3} \mathrm{keV}^{-1} \mathrm{~kg}^{-1} \mathrm{yr}^{-1}$ [13]15] observed with the EXO-200 detector, around the double beta decay Q-value of $Q_{\beta \beta}=2457.83 \pm 0.37 \mathrm{keV}$ [16], is one of the lowest in its field. A detailed background analysis has been published in Ref. [17]. This analysis compared the data-derived estimates of the activity contents of detector components with those obtained in the radioassay program. In an alternate approach the radio-assay values were fed into the detector simulation to arrive at expectation values. Detector background predictions which were made before data-taking agree reasonably well with the observed rate. It was further noted that for most components the radioassay program yielded stronger constraints than the data driven analysis [17. The EXO-200 detector thus provides some validation of the data, methods and assumptions reported in this work and the previous EXO-200 component radioactivity compilation [2].

The EXO-200 materials analysis effort was structured around a detailed, GEANT 3.21 based Monte Carlo simulation of the experiment. A total background budget of 33 events per year in $110 \mathrm{~kg}$ of xenon (after cuts) for events in the energy interval $Q_{\beta \beta} \pm 2 \sigma_{\beta \beta}$ was defined [11], where $\sigma_{\beta \beta}$ stands for the energy resolution at the Q-value. A target value of $\sigma_{\beta \beta} / Q_{\beta \beta}=0.015$ was chosen. Major experiment components, such as the cryostat or the lead shield, were allowed to contribute $10 \%$ of the total budget while small components were given a $1 \%$ background allowance. This fuzzy scheme allowed material acceptance decisions to be made before all components had been specified and analyzed for their radioactivity content. The background allowance was then translated into a maximally allowable radioactivity content for each component by means of the Monte Carlo model. This allowance determined, in turn, the choice of analysis method. All materials and components used during the EXO-200 construction were subject to this process; no exceptions were made. 
The results of the EXO-200 radioactivity screening program are reported as element concentrations, in units of $\mathrm{g} / \mathrm{g}$ (grams of impurity per gram of sample). Multiplication with conversion factors of $3.17 \cdot 10^{4}(\mathrm{~Bq} / \mathrm{kg}) /(\mathrm{g} / \mathrm{g})\left({ }^{40} \mathrm{~K}\right), 4.07$. $10^{6}(\mathrm{~Bq} / \mathrm{kg}) /(\mathrm{g} / \mathrm{g})\left({ }^{232} \mathrm{Th}\right)$ and $1.23 \cdot 10^{7}(\mathrm{~Bq} / \mathrm{kg}) /(\mathrm{g} / \mathrm{g})\left({ }^{238} \mathrm{U}\right)$ yields nuclide specific activities in units of $\mathrm{Bq} / \mathrm{kg}$.

The following analysis methods, described below, were employed in this work and in the previous measurement campaign:

(1) Above-ground and below-ground low-background gamma-ray spectroscopy using Ge detectors.

(2) Glow-discharge mass spectroscopy (GDMS)

(3) Inductively Coupled Plasma Mass Spectroscopy (ICPMS)

(4) Neutron Activation Analysis (NAA)

The radioactivity-induced background for the EXO-200 search for neutrinoless double beta decay stems, to a large extent, from ${ }^{208} \mathrm{Tl}$ (Th-series) and ${ }^{214} \mathrm{Bi}$ (U-series) $\beta+\gamma$ decays. ${ }^{60} \mathrm{Co}$ coincidence summing and cosmogenic activation play a small role too. Germanium counting has the lowest analysis sensitivity but determines the relevant ${ }^{208} \mathrm{Tl}$ and ${ }^{214} \mathrm{Bi}$ decay rates directly. No further assumptions are needed to convert radioactivity concentrations into background event rates. GDMS, ICPMS and NAA offer improved sensitivity; however, they determine the concentrations of the long-lived heads of the decay series, ${ }^{232} \mathrm{Th}$ and ${ }^{238} \mathrm{U}$, respectively. The Th and $\mathrm{U}$ decay rates can only be related to those of ${ }^{208} \mathrm{Tl}$ and ${ }^{214} \mathrm{Bi}$ by making assumptions about the establishment of chain equilibrium. For the EXO-200 preparation, for results reported in Ref. [2], and for results reported here, it was decided to assume that the decay chains are in secular equilibrium. The fact that background estimates made prior to data taking agree reasonably well with the EXO-200 observation ultimately justified this assumption. Improved analysis sensitivity therefore comes at the expense of reliance on assumptions. In a sense, one risk has to be balanced against another.

\section{Underground Gamma Counting}

All of the gamma counting was done at the Vue-des-Alpes laboratory, located in a lateral cavern in the Vue-des-Alpes road tunnel, in the Swiss Jura. Some measurements were carried out above ground with two Ge detectors used also for NAA. They are described in Section 5.2. The vertical rock overburden is $230 \mathrm{~m}$, corresponding to 600 mwe (see Refs. 2,18]). The rate of cosmic muons through a flat horizontal surface is around $0.2 \mathrm{~m}^{-2} \mathrm{~s}^{-1}$, about a factor 1000 less than above ground. Cosmic-ray neutrons are completely eliminated. A Ge detector made by Eurisys (now Canberra) in 2001 was used. It is a p-type 
coaxial device. The volume of the germanium crystal is $400 \mathrm{~cm}^{3}$. The active volume is reduced by a dead layer of a few times $100 \mu \mathrm{m}$ on the outer side and the top. The dead layer on the inner hole is negligible. The crystal is housed in a can-shaped vessel forming the end of a cryostat and made from ultra-clean Péchiney aluminum with relatively low Z. The end cap is particularly thin $(0.5 \mathrm{~mm})$, providing a good $\gamma$ transmission even at low energy. The germanium crystal is mounted on a copper cold finger, cooled by liquid nitrogen.

The detector is surrounded by a shield made from electrolytic tough pitch (ETP) copper inside, with a thickness varying from $12.5 \mathrm{~cm}$ to $20 \mathrm{~cm}$, and by $15 \mathrm{~cm}$ to $20 \mathrm{~cm}$ of lead outside. This efficiently suppresses local $\gamma$ activities. The shielding rests on a steel table. The liquid nitrogen dewar is located outside, and the cold finger traverses the shielding. The shielding copper was taken over and adapted from a Ge experiment in the Gothard tunnel [19]. The lead was purchased new. Both the copper and lead were originally obtained from local companies and samples of both had been tested with an older Ge detector with a sensitivity of order $10 \mathrm{ng} / \mathrm{g}$ to $\mathrm{U}$ and $\mathrm{Th}$.

The radon level in the lab was measured to fluctuate around $85 \mathrm{~Bq} / \mathrm{m}^{3}$. To suppress the background from ${ }^{222} \mathrm{Rn}$, the shielding itself is enclosed in an aluminum box resting on the steel table. The box is airtight. It is over-pressurized with boil-off nitrogen from an external dewar, also used to refill the germanium dewar. The top of the aluminium box and the top of the shielding can be opened with a hoist to insert samples into a cavity surrounding the detector.

The energy resolution is $1.4 \mathrm{keV}$ FWHM at $238 \mathrm{keV}$, and $2.5 \mathrm{keV}$ at $1460 \mathrm{keV}$, scaling with the the square root of the energy above that. At low energy the response is nearly Gaussian, while a low-energy tail appears at higher energies.

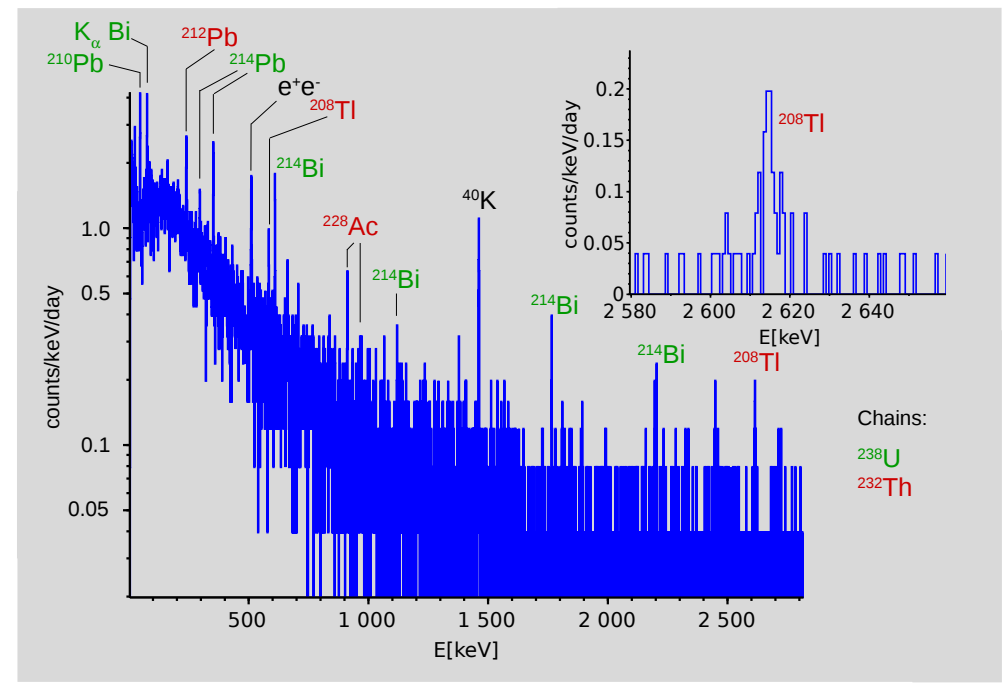

Fig. 1. The background spectrum of the Ge counter at the Vue-des-Alpes laboratory, accumulated during $882.5 \mathrm{~h}$. 
Table 1

\begin{tabular}{l|r|r|l|c} 
chain & nuclide & $\mathrm{E}_{\gamma}(\mathrm{keV})$ & \multicolumn{1}{|c}{ BR } & Background $\left[\mathrm{d}^{-1}\right]$ \\
\hline${ }^{238} \mathrm{U}$ & ${ }^{214} \mathrm{~Pb}$ & 295.20 & 0.185 & $1.91 \pm 0.33$ \\
& ${ }^{214} \mathrm{~Pb}$ & 351.92 & 0.358 & $3.25 \pm 0.39$ \\
& ${ }^{214} \mathrm{Bi}$ & 609.30 & 0.448 & $2.87 \pm 0.34$ \\
& ${ }^{214} \mathrm{Bi}$ & 1120.28 & 0.148 & $0.64 \pm 0.17$ \\
& ${ }^{214} \mathrm{Bi}$ & 1764.50 & 0.154 & $0.69 \pm 0.16$ \\
\hline \multirow{6}{*}{${ }^{232} \mathrm{Th}$} & ${ }^{210} \mathrm{~Pb}$ & 46.54 & 0.0425 & $4.17 \pm 0.47$ \\
& ${ }^{228} \mathrm{Ac}$ & 338.30 & 0.113 & $0.45 \pm 0.25$ \\
& ${ }^{228} \mathrm{Ac}$ & 911.20 & 0.266 & $0.66 \pm 0.19$ \\
& ${ }^{228} \mathrm{Ac}$ & 968.97 & 0.162 & $0.34 \pm 0.14$ \\
& ${ }^{212} \mathrm{~Pb}$ & 238.63 & 0.433 & $4.07 \pm 0.46$ \\
& ${ }^{208} \mathrm{Tl}$ & 583.19 & 0.3055 & $1.13 \pm 0.24$ \\
& ${ }^{208} \mathrm{Tl}$ & 2614.53 & 0.3585 & $0.72 \pm 0.15$ \\
\hline & ${ }^{40} \mathrm{~K}$ & 1460.86 & 0.1066 & $3.00 \pm 0.30$ \\
\hline & ${ }^{137} \mathrm{Cs}$ & 661.60 & 0.851 & $0.99 \pm 0.23$ \\
\hline & ${ }^{60} \mathrm{Co}$ & 1173.23 & 0.9999 & $0.07 \pm 0.12$ \\
& ${ }^{60} \mathrm{Co}$ & 1332.50 & 0.9998 & $0.11 \pm 0.11$ \\
\hline
\end{tabular}

List of the lines with energy $\mathrm{E}_{\gamma}$ and branching ratio BR [20] considered in the routine analysis of samples. The nuclides within a chain are arranged in chronological order. The internal background of the Vue-des-Alpes detector is also given.

\subsection{Standard operations}

Depending on their nature, samples were cleaned before testing. Metallic samples were wiped with acetone and cleaned in an ultrasonic bath with methanol only. Samples with plastic pieces were wiped with methanol. Samples were usually packed in plastic bags or plastic vials to avoid contaminating the shielding. This work was done in a clean room. Samples were inserted into the cavity surrounding the detector, where they could be placed in a geometry optimizing the acceptance. The first 24 hours of data after closing the shielding was discarded to make sure that all the radon contamination had been purged out. Measurements usually lasted one week, but were extended to up to four weeks depending on the activity found and the required sensitivity. 


\subsection{Count rates}

The count rates of the most relevant lines were obtained by integrating the counts in a window with a width of 2 FWHM. The background under the line was extrapolated from a window 4 to 8 times as wide and was subtracted.

The lines with energy $\mathrm{E}_{\gamma}$ and branching ratio $\mathrm{BR}$ [20] given in table 1 were analyzed routinely. They include the main lines in the ${ }^{238} \mathrm{U}$ and ${ }^{232} \mathrm{Th}$ chains, the lines resulting from ${ }^{40} \mathrm{~K},{ }^{137} \mathrm{Cs}$, and ${ }^{60} \mathrm{Co}$ decays.

\subsection{The internal background}

The background from internal activities, or originating from the shielding, was measured at regular intervals without any sample, and was observed to be fairly constant. The integral rate from $5 \mathrm{keV}$ to $2.8 \mathrm{MeV}$ was $26.6 \pm 0.17 \mathrm{~h}^{-1}$. The spectrum is displayed in Fig. 1. The ${ }^{40} \mathrm{~K}$ line dominates, but weak lines from the ${ }^{238} \mathrm{U},{ }^{232} \mathrm{Th}$ chains are also visible. The count rates are listed in Table 1.

The continuum is largely due to Compton scattering accompanying the observed $\gamma$ transitions, but also in part to direct cosmic $\mu$ hits and to bremsstrahlung generated by these $\mu$ 's in the lead and copper shielding. The background spectrum was analyzed with the same procedure as that for the samples. The rates obtained for each of the lines listed in Table 1 was taken as background, and subtracted from the rates with a given sample, yielding the final count rate attributed to that sample. All uncertainties were added in quadrature.

\subsection{Evaluation of the activity}

The inspection of the integral count rate from $5 \mathrm{keV}$ to $2.8 \mathrm{MeV}$ provides a first check of the radiopurity of a sample. Any excess above the background value indicates a contamination. The analysis of the transitions mentioned above allowed more specificity. The detector acceptance as a function of energy was calculated with GEANT3 for each sample geometry and chemical composition.

For ${ }^{40} \mathrm{~K}$ and ${ }^{137} \mathrm{Cs}$ only one transition is available, and the activity is obviously that obtained from the counts in the corresponding peak. For ${ }^{60} \mathrm{Co}$, the average from the two transitions at 1173 and $1332 \mathrm{keV}$ was computed. For the ${ }^{238} \mathrm{U}$ and ${ }^{232}$ Th chains, if the data were consistent with secular equilibrium, the weighted average of all the transitions in Table 1 was calculated. One exception was the $46.5 \mathrm{keV}$ transition in ${ }^{210} \mathrm{~Pb}$ in the ${ }^{238} \mathrm{U}$ chain, which was not included. 
It gives in general a comparatively poor precision, because of low acceptance. Sometimes the secular equilibrium appears broken at that level. ${ }^{210} \mathrm{~Pb}$ is listed separately.

If an activity was observed above background at the $90 \%$ confidence level for any of the mentioned nuclides, the nuclide was declared active. In all other cases an upper limit was calculated using the renormalized Gaussian method, which takes into account that the true activity cannot be negative.

The probability distribution for the true activity is taken as a Gaussian function centered at the measured activity. The statistical uncertainty at the $68 \%$ confidence level of the measured activity was taken as the standard deviation. Only the parameter space corresponding to true activities larger or equal to zero is retained, and the Gaussian function is normalized to 1 in that parameter space. To calculate the limit, the probability distribution is integrated from zero upward, until the fractional area corresponding to the desired confidence level is reached.

The quoted activities for ${ }^{238} \mathrm{U}$ and ${ }^{232} \mathrm{Th}$ are effective activities, assuming secular equilibrium. The real values may differ, but the effective activities are a precise measure of the penetrating $\gamma$ backgrounds, the most worrisome type of backgrounds for materials outside of the sensitive medium itself. The $\alpha$ and $\beta$ emissions from outer materials are mostly blocked from reaching the sensitive volume.

\section{GDMS}

Glow Discharge Mass Spectrometry (GDMS) is one of the most comprehensive trace element analysis techniques currently available for the direct determination of the composition of conductive solid materials. This is especially important for high purity materials where low detection limits are desirable. Since samples are analyzed in solid form, laborious and error-prone dissolution procedures inherent to such techniques as Inductively Coupled Plasma Mass Spectrometry are avoided.

In GDMS, the sample functions as the cathode, whereas the reaction cell forms the anode portion of this two electrode system. A low flow of pure argon sustains a DC electrical discharge at low pressure in which sputtering of the sample occurs. Application of several hundred volts between the electrodes establishes the discharge, producing a low-pressure plasma containing electrons and Ar ions. The major voltage drop occurs close to the sample cathode and leads to the sputtering of the surface by bombardment with energetic Ar ions. Atoms sputtered from the surface enter the plasma wherein they are rapidly 
ionized by a number of processes, including collisions with energetic electrons as well as Penning ionization. One of the principal advantages of the GD technique is that it allows the determination of the bulk composition of the sample, assuming intrinsic homogeneity. The ionized atoms are then extracted into the mass spectrometer for separation based upon their mass/charge ratio followed by detection.

Under the typical discharge conditions, with the Thermo Fisher VG 9000 GDMS instrument used for our work, the surface of the sample is ablated at a rate of roughly $1 \mu \mathrm{m} / \mathrm{min}$. It should also be noted that all samples are chemically pre-cleaned prior to analysis to remove any surface contamination that may have occurred during the cutting/shaping processing of a sample into a pin-shaped geometry suitable for the instrument. The sample is then sputtered for up to 30 minutes prior to acquisition of analytical data, to ensure that all possible surface contamination has been removed. Analyte concentrations are obtained as the ratio of the ion current from each impurity detected in the sample to that from the sample matrix (the "matrix" refers to the primary or overall composition of the sample, as opposed to individual trace impurities). These data are then corrected for minor element dependent changes in relative sputter yields using relative sensitivity factors (RSF). The latter are determined by pre-calibrating the instrument using reference material of known elemental composition. Results are typically valid within a factor of two for all elements except $\mathrm{C}, \mathrm{N}$ and $\mathrm{O}$, for which a factor of five is typical. However, these elements are not relevant for the current work.

A unique feature of GDMS is that these RSF values, although instrument specific, do not change over time, are stable for years and matrix effects are low enough such that semi-quantitative, order-of-magnitude or better measurements can be made even without matrix-matched calibration standards.

The high sensitivity of this analytical technique enables determination of impurities in the sample at the order of $10 \mathrm{pg} / \mathrm{g}$ if using a sufficient analysis time (high number of mass scans). Also, the universality of the method allows for the detection of almost all elements in the periodic table in conductive inorganic matrices.

A potential drawback of GDMS is that it is a micro-sampling technique requiring a solid sample and, as such, is subject to the homogeneity of that portion of the sample sputtered into the ionization volume. As sample inhomogeneity cannot be accounted for, it may contribute to a substantial increase in the reported estimated uncertainties. As there are very few reference materials available for high purity metals and semiconductor materials, the uncertainty associated with the RSF correction factors can be significant. These facts inherently limit the accuracy and relegate the method, for our purposes, to a semi-quantitative technique. 


\section{ICPMS}

Inductively Coupled Plasma Mass Spectrometry (ICPMS) is one of the most powerful analytical methods for trace and ultra-trace analysis, offering sub $\mathrm{pg} / \mathrm{g}$ detection limits for $\mathrm{U}$ and Th with minimal analysis time. Samples, most commonly in the form of liquid solutions, are introduced into an argon gas stream. The resulting aerosol mixture is ionized in an RF field, creating a plasma. Molecules are dissociated, and most elements are ionized with high efficiency. Ions are extracted electrostatically into a mass spectrometer. The technique offers higher sensitivity than GDMS, and the liquid sample form allows for easy preparation of calibrated standards or spiked sample solutions. This allows calibrations to be performed in identical conditions to the sample measurements, thus providing highly quantitative results.

The clear difficulty for ICPMS is the need to provide the sample in a suitable liquid form, typically aqueous. This can sometimes require development of specialized dissolution techniques using strong, high-purity acids and, for more difficult samples, specialized equipment as well such as a microwave digestion system, asher, etc. Metals are typically the easiest samples to dissolve in acids, but unlike for GDMS, non-conductive samples can be analyzed if suitable dissolution techniques exist. While the instruments can measure concentrations in liquid from a few $\mathrm{fg} / \mathrm{g}$ up to hundreds of $\mu \mathrm{g} / \mathrm{g}$, the sensitivity of the technique is generally limited by the need to keep the total dissolved solid concentration low, typically far below $1 \%$. High matrix levels give rise to deposition of matrix constituents on the surface of the interface cones (metal orifices where ions pass from high pressure plasma to low pressure spectrometer), causing significant signal drift. Once the samples are in a liquid form, it is also possible to enhance sensitivities by developing techniques to pre-concentrate the elements of interest relative to the matrix. Analyte separation and pre-concentration has become of paramount importance in order to achieve sub-pg/g level analysis. However, all chemicals used in sample processing must be carefully controlled to avoid introduction of background contaminants. Analysis blanks must be subtracted, producing systematic uncertainties which are often a limiting factor for improvements in sensitivity.

For the work reported here we followed sample digestion by separation of analytes from the matrix. This can be achieved by passing the digested sample through a chemical filter which can separate the analytes from the matrix. Specifically we used UTEVA resins from Eichrom Industries, Inc. consisting of diamyl amylphosphonate (DAAP) extractant adsorbed onto an inert polyacrylamide support consisting of particles having an external diameter of 100$150 \mu \mathrm{m}$ [21] and packed into chromatographic columns. The analytes are initially captured by the extractant while the matrix passes through. Uranium can than be eluted from the column with $0.02 \mathrm{M} \mathrm{HCl}$, and $0.5 \%$ oxalic acid 
was required and used in order to elute Th. Both fractions were evaporated to near dryness; the Th fraction was further decomposed with a 1:1 mixture of concentrated $\mathrm{HNO}_{3}$ and $30 \% \mathrm{H}_{2} \mathrm{O}_{2}$, once again evaporated to near dryness and reconstituted to $2 \mathrm{ml}$ with $0.5 \%$ nitric acid. This extra step was necessary to decompose organic extractants from the column to achieve a stable detection efficiency, thereby further elevating the blank.

All steps of this procedure need to be monitored closely in order to ensure accurate results. Purity of the chemicals, materials and reagents used in the analysis must also be verified. Acids used for the dissolution and separation procedures were of super-pure analytical grade (TAMA PureAA-100 or higher) All lab-ware used was cleaned with nitric acid for at least $24 \mathrm{~h}$. Sample digestion and cleaning procedures were conducted in a class 100 clean room. Separations were undertaken in a class 10 fume hood. Blanks included all reagents and were run in parallel with samples. New resins exhibited Th concentrations of approximately $8 \mathrm{pg} / \mathrm{g}$. After thorough cleaning with several alternate washings with $\mathrm{HCl}$, oxalic acid and double-distilled water (DDW), thorium concentration levels were reduced to about $1.5 \mathrm{pg} / \mathrm{g}$. The recovery efficiencies for $\mathrm{U}$ and $\mathrm{Th}$ were approximately $80 \%$ and $60 \%$ respectively. A typical initial sample mass of $1 \mathrm{~g}$ represented by a $2 \mathrm{ml}$ reconstituted volume results in an effective sample-to-solution ratios of roughly $35 \%$ by mass. In comparison, a direct digestion measurement without the separation step is typically performed at concentrations of around $0.1 \%$ dissolved solid, and typically, for the Perkin Elmer Elan-DRC II used for this work, can achieve limits of detection (LODs) for $\mathrm{U}$ and Th of about $15 \mathrm{ppt}$ in a solid sample. The pre-concentration step represents about a 350 times signal enhancement, potentially resulting in LOD's as low as $45 \mathrm{fg} / \mathrm{g}$. The actual LOD's achieved are however significantly limited by the previously mentioned backgrounds arising from the resins and reagents, and to some extent by the small reconstituted solution volume. We note that since these data were taken, further development of this basic method has led to detection limits at the level of $10 \mathrm{fg} / \mathrm{g}$, achieved by improving the background levels [22].

Procedural LODs of about 0.5 and $1.5 \mathrm{pg} / \mathrm{g}$ in solids for $\mathrm{U}$ and Th respectively were achieved, based on the dissolution of $1 \mathrm{~g}$ samples and final reconstitution to $2 \mathrm{ml}$. Prior to digestion of a sample, a surface cleaning procedure was performed in order to remove any impurities present on the surface of the sample. This was achieved by placing the sample into acid solutions for a period of time. The acid and strength used, as well as the time period, depended on the matrix of the sample. For example, surface cleaning for $\mathrm{Cu}$ matrix samples were performed by placing the sample into a $3 \mathrm{M} \mathrm{HNO}_{3}$ solution for 20 minutes; aqua regia was used for $\mathrm{Al}$.

Thorium showed a severe memory effect during the determination by ICPMS as it tends to adhere to the walls of the pump tubing, spray chamber and 
cones, making longer washing times necessary between samples or standards. Also, low recoveries for Th were observed when using a Pyrex beaker during the evaporation step. This was overcome by using Teflon beakers instead.

\section{NAA}

Neutron activation analysis (NAA) is a well-established trace element analysis technique. It is based on the capture of thermal and epi-thermal neutrons by a stable or meta-stable nuclear species. In case the newly created nuclear species has a shorter half-life than the mother, the decay rate is boosted. This works if a sufficient fraction of the target nuclei is transmuted. Depending on the capture cross section (and thus nuclear species), this requires the use of high neutron-flux research reactors to achieve an enhancement in the decay rate. It is applicable to materials where the matrix, does not form long lived radioactivity after neutron capture. Many of the analysis details were discussed in our previous paper [2] and will not be repeated. We will, instead, provide a more detailed discussion of the procedures used to derive elemental concentrations from measured $\gamma$-ray spectra.

The activations reported here utilized the MIT Reactor (MITR) as neutron source. Sample preparation and counting were done at EXO labs. MITR is a $6 \mathrm{MW}$ tank-type reactor, utilizing highly enriched uranium fuel, resulting in a compact fuel assembly. It has a light-water moderator and heavy-water and graphite neutron reflectors. The activations were performed at reactor powers varying between 4.8 and 5.8 MW. MITR has two pneumatic sample delivery systems. One with 2" diameter (2PH1), offering high thermal and fast neutron fluxes (up to $5.5 \cdot 10^{13} \mathrm{~cm}^{-2} \mathrm{~s}^{-1}$ observed in our studies). A second tube with 1" diameter (1PH1) shows lower thermal neutron flux and a much reduced fast neutron flux. We utilized 1PH1 for samples with low metal content to reduce background caused by fast-neutron induced side reactions. All work presented here is based on irradiations in 2PH1. Both insertion tubes are spatially located in the reflector region of the reactor. The neutron spectrum is similar to that of a light water reactor. Its parametrization is discussed later in this paper.

NAA usually utilizes radiative neutron capture on some target element of interest $T$, forming the unstable daughter species $D: T(n, \gamma) D$. For activation products with sufficiently long life times, $\tau_{D}$, the delayed beta decay of $D$ can be measured, for example by utilizing resultant $\gamma$-radiation and high resolution

Ge-detectors. The irradiation of a sample, containing $N_{T}$ atoms of the species of interest and lasting the time $t_{i}$, results in a time $t$ dependent nuclear decay 
rate $R_{D}$ of the activation product:

$$
R_{D}(t)=N_{T} \cdot\left(1-e^{-t_{i} / \tau_{D}}\right) \cdot e^{-t / \tau_{D}} \cdot \int_{0}^{\infty} \Phi(E) \cdot \sigma(E) d E
$$

where $\Phi(E)$ denotes the energy dependent neutron flux the sample is exposed to while inside the reactor, $\sigma(E)$ is the differential neutron capture cross section, and $t=0$ is chosen as the end of the irradiation. Equation 1 holds for irradiations consuming only a small fraction of the target atoms. Equation 1 can be used to derive $N_{T}$ (the quantity of interest) from a measured decay rate of the activation daughter. This requires calculation of the reaction yields per atom given by the integral in Equation 1, and of the time corrections (the exponential factors in Equation 1). Appendix $\mathrm{A}$ of this article describes the procedures used to determine the yields from tabulated cross sections and measured neutron fluxes.

Application of this analysis tool allowed to routinely reach Th/U sensitivities of $\mathrm{pg} / \mathrm{g}$ level or bettter.

\subsection{Sample preparation and recovery}

Both sample preparation and recovery followed a well-defined protocol. This is important for achieving reproducible results. NAA results are typically reported as element concentrations per unit sample mass. This normalization assumes that the impurities are dominantly located in the sample bulk. Special attention therefore needs to be given to the removal of any surface impurities before sample analysis. Before activation the samples were handled in a class 500 clean room. The sample surfaces were cleaned with DI water, organic solvents and dilute $\mathrm{HNO}_{3}$, all analytical grade or better. All beakers used in this process were pre-cleaned using the same sequence of solvents. After the cleaning and for activations of less than $10 \mathrm{~h}$ duration, the samples were packed in $5 \mathrm{ml}$ polyethylene (PE) bottles (cleaned in $5 \mathrm{ml}$ solvent and acid) that were then sealed using a soldering iron. Leak tightness of the containers was verified in a heated water bath. For long term activations samples were sealed into cleaned quartz containers. All samples and their containers were carefully weighed using an analytic balance to provide the normalization and to quantify the post-activation sample recovery fraction. The fact that sub-pg/g sensitivities are routinely reached, even for small samples, verifies the validity of these cleaning procedures.

After activation, the handling focused on the clean separation of the sample from the, often externally contaminated, sample bottle. A separate glove box was used. PE bottles were softened during the activation. This limits the activation time at MITR to about $10 \mathrm{~h}$, after which these bottles tend to 
disintegrate, leading to sample loss. Sample removal was achieved by carefully cutting off the top of the bottle. The sample was then removed by pouring it into a clean PE counting bottle or retrieving it with tweezers. Care was needed so as not to transfer any of the abundant contamination on the outside of the counting bottle onto the sample. All beakers and instruments were only used once to avoid any carry-over problems. After recovery the samples were weighed.

Recovering activated samples from quartz vials requires a different procedure. Because of their mechanical strength, quartz vials need to be cracked inside a metal tube (to prevent explosive pressure relief). As this procedure does not allow to separate the sample from the bottle's external surfaces, the bottles needed to be vigorously cleaned with solvents and acids before cracking. The cleaning agents were counted after each cleaning, the bottles were cracked after no more activity was detected.

Depending on the activated sample, recovery can hindered if the sample decomposes during activation. In this case, sometimes only a fraction of the sample can be recovered cleanly and safely.

\subsection{Gamma-ray fitting}

The activated samples are counted on three high resolution Ge detectors, commencing on average 33 hours after the end of irradiation. Gamma-ray spectra are accumulated initially over $1 \mathrm{~h}$ time intervals. As time goes on and the short-lived activities die out, longer and longer accumulation times are chosen to obtain reasonable statistics in each run. This allows following the decay of the unstable nuclides. A measurement of the half-life constitutes an additional observable. Gamma-ray peak energies $E_{\gamma}$ determine the decaying parent nuclide $j$. Fitting peak $k$, the integral $I\left(E_{\gamma, k}, t_{i}\right)$, determined at time $t_{i}$ (combined with gamma energy dependent counting efficiency $\varepsilon\left(E_{\gamma, k}\right)$ branching ratio $b_{k, j}$ and run counting time $t_{c i}$ ) provides an estimate of the activity $A_{k, j}\left(t_{i}\right)$ of the parent nuclide and its error $\sigma_{A_{k, j}}\left(t_{i}\right)$ :

$$
A_{k, j}\left(t_{i}\right)=\frac{I\left(E_{\gamma, k}, t_{i}\right)}{t_{c i} \cdot \varepsilon\left(E_{\gamma, k}\right) \cdot b_{k, j}}
$$

A custom peak fitting code called GDFIT performs a coupled fit to all $\gamma$ lines associated with a particular nuclide and thus summarizes them into one nuclide-specific activity. More detail is provided in Ref. [2]. The $\chi^{2}-$ minimizations are based on the CERN software package MINUIT. This procedure is essentially equivalent to performing a weighted average of all single gamma-line activities, minimizing the counting error. $\varepsilon\left(E_{\gamma, k}\right)$ is determined 
using a calibrated mixed nuclide solution for various counting geometries. Analysis consistency is routinely verified by counting activated samples on all three of the available detectors and comparing the derived sample activities.

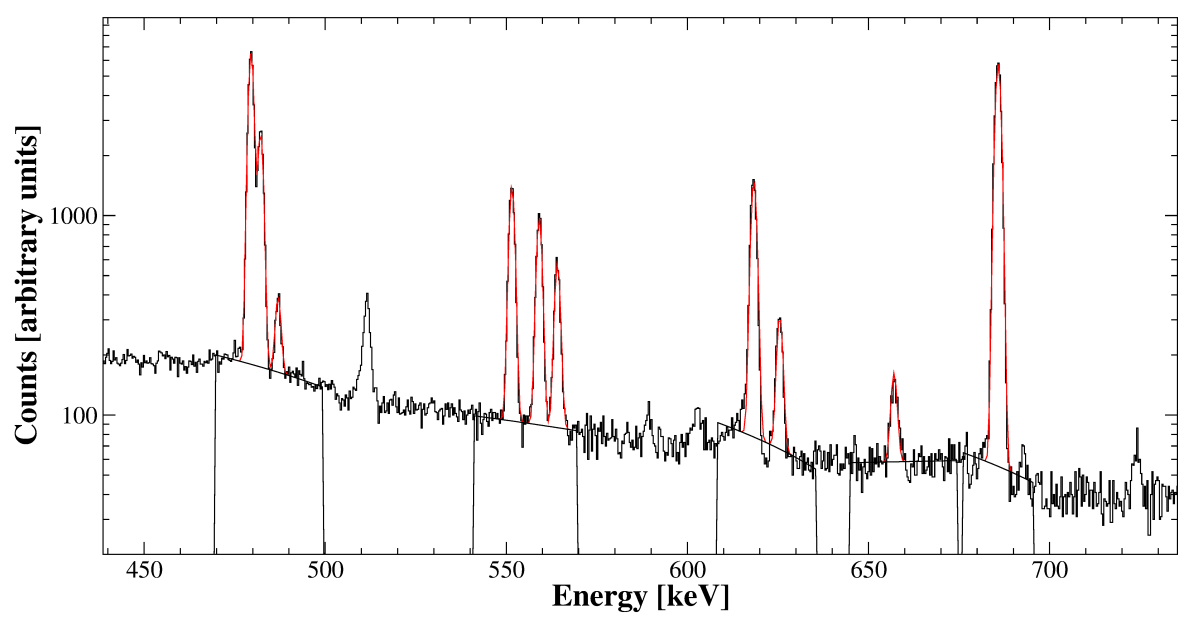

Fig. 2. Example for a simultaneous gamma line fit performed for the 480, 552, 618, 626 and $686 \mathrm{keV}$ lines of ${ }^{187} \mathrm{~W}$, the 559 and $657 \mathrm{keV}$ lines of ${ }^{76} \mathrm{As}$ and the 482,489 and $564 \mathrm{keV}$ lines of ${ }^{181} \mathrm{Hf},{ }^{47} \mathrm{Ca},{ }^{122} \mathrm{Sb}$, respectively.

Because of the multitude of peaks present in any of the spectra, care has to be taken to include in the fit gamma lines of similar energy to, but not associated with, the activity of interest. These can produce peak area degeneracy and/or can influence the background fit if not properly included. For multiple-gamma activities, constraints through the linked branching ratios can help significantly to resolve such degeneracies. If the interfering decay also has multiple peaks then even degeneracy of perfectly overlapping peaks can be resolved while still providing added statistical benefit for the activity of interest. Interferences that are not properly fit can often be spotted as a deviation of the half-life from its expectation value. In the worst case scenario, degeneracy can be resolved by fitting multiple half-life components to the time series. In principle this could be included as part of a single global fit to peaks at all time intervals, but this was not implemented and could have practical challenges.

All gamma peaks of interest are fitted in all $\gamma$-spectra and then collected as a function of time. The various reported nuclide activities are plotted as a function of time, as shown in Figure 3. The fit to the time dependence of the activities is compared to tabulated half-lives, further enhancing analysis selectivity. Once a nuclide assignment has been made the activity at some reference time is re-fit by fixing the half-life to its tabulated values as shown in Figure 3. In this way all measurements contribute to a single activity value. For high statistics data, as shown in the left panel of Figure 3, the data shows fluctuations beyond the statistical errors. These are due to small differences in the placement of the sample on the Ge detector and hence the counting solid angle, when the measurements of multiple samples necessitated the removal 


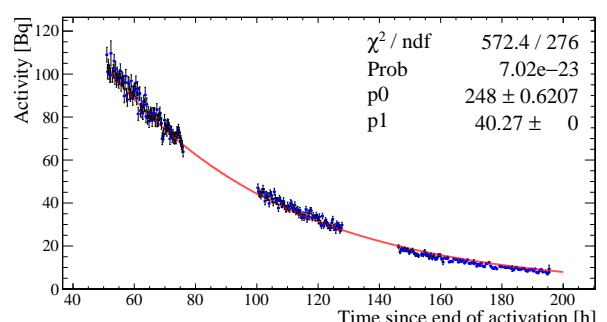

(a)

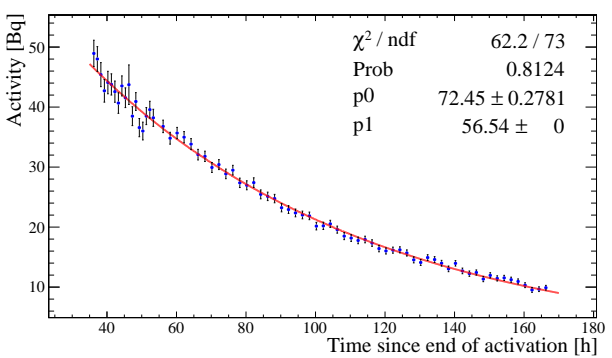

(b)

Fig. 3. Time dependent ${ }^{140} \mathrm{La}$ (left) and ${ }^{239} \mathrm{~Np}$ (right) activities, derived from repeated analysis of gamma lines. The half-lives have been fixed to their tabulated values. The left panel shows data derived from interrupted counting of the same sample to allow counting of several samples on one detector. Repeated reinsertion of the sample results in an imperfect reproduction of the counting solid angle, visibly impacting the fit quality. The right panel shows data derived from uninterrupted counting.

and later re-insertion of a particular sample. A point-by-point $10 \%$ systematic error is added to the activities to account for this variability.

\section{Data and Conclusions}

EXO-200 has produced one of the lowest-background particle-interaction detectors ever built, in large part because of the exhaustive effort to screen construction materials for trace levels of radioactive contaminants. We conclude our reporting of the results of these studies with many newly measured materials or, in some cases, re-confirmed measurements of new materials batches. Results are reported in Table 2. Combined with the previous work [2], we have established an extensive library of material radio-purities suitable for use in planning the detailed design of new, next-generation detectors. 


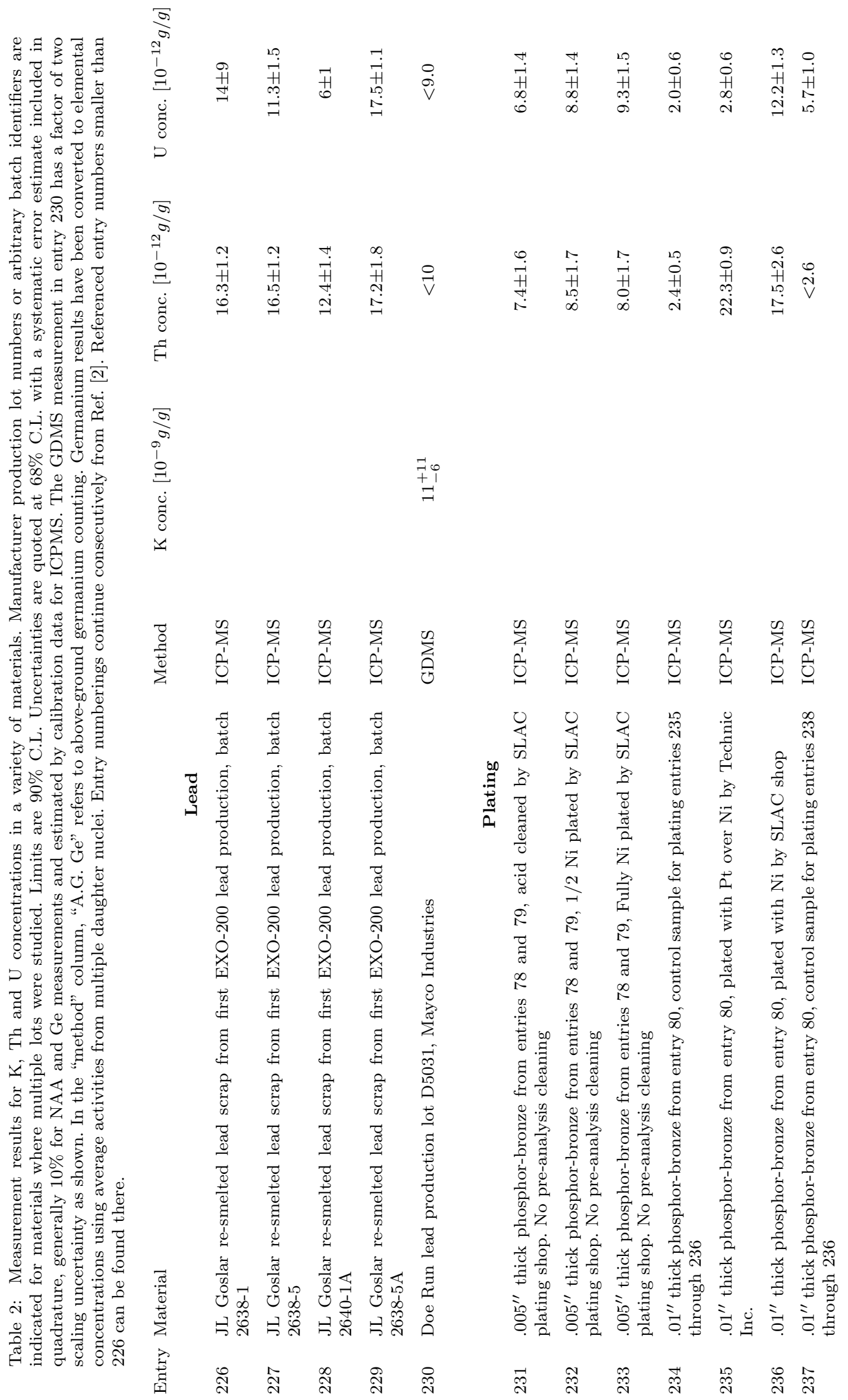




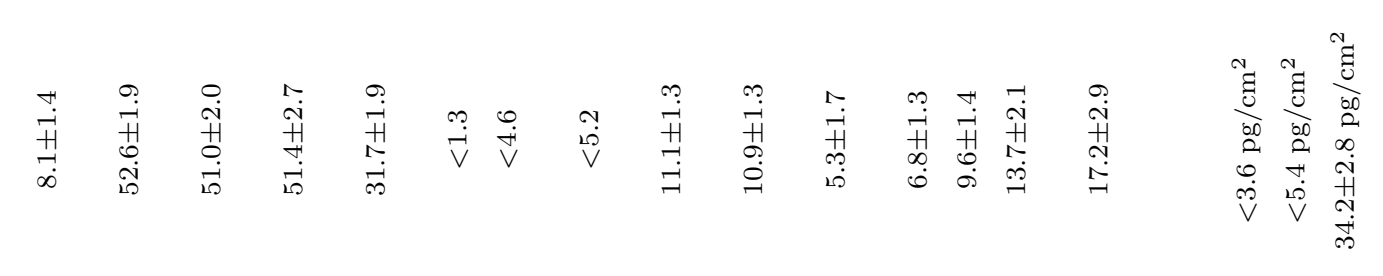

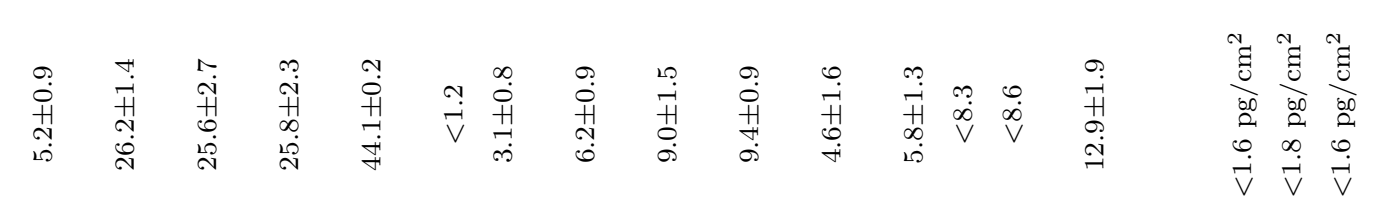

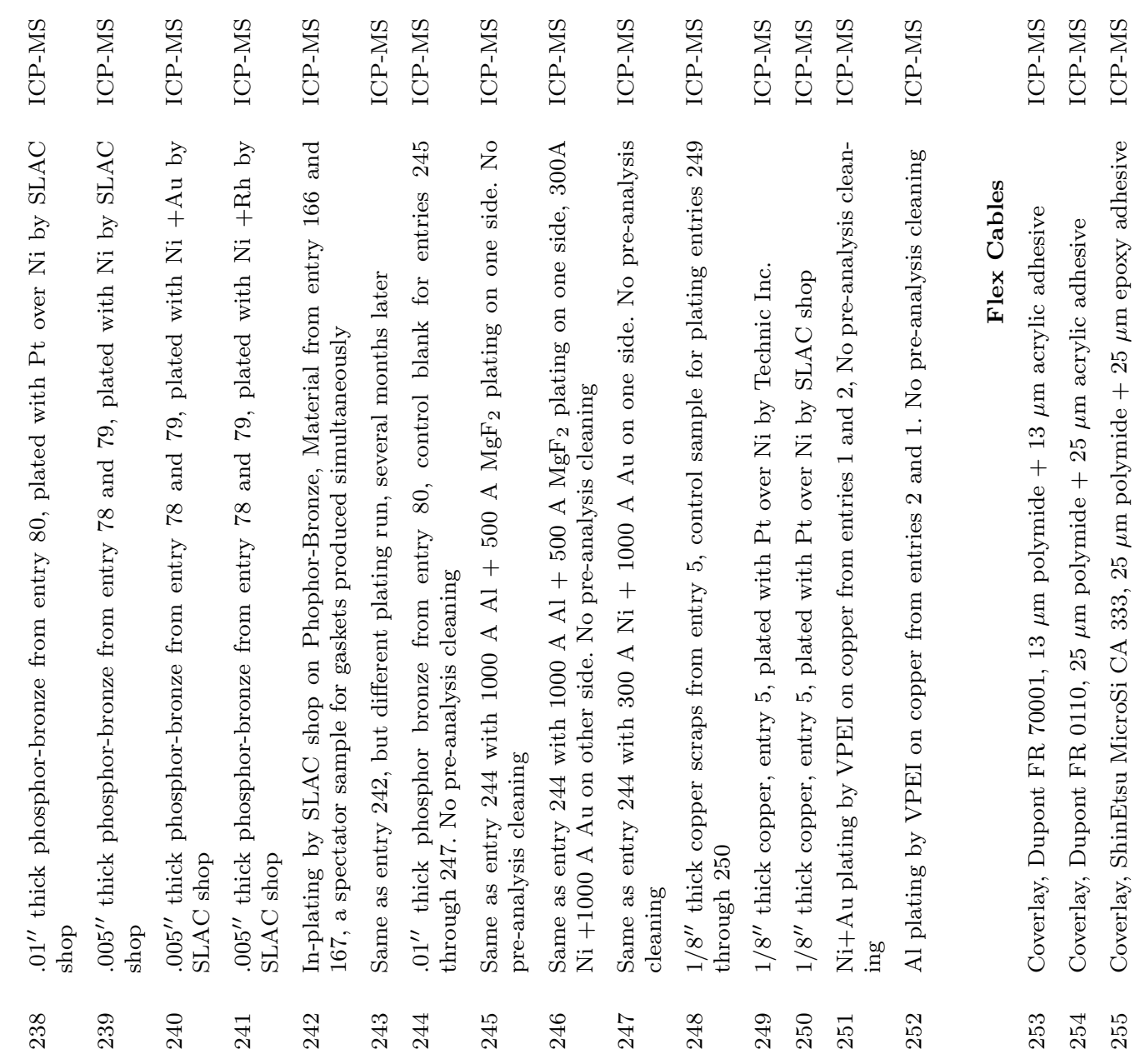




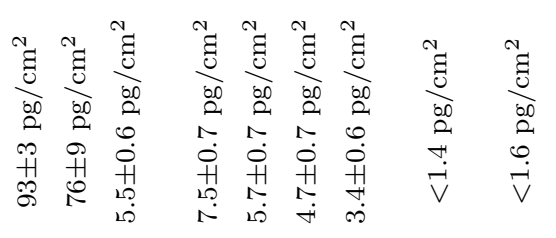

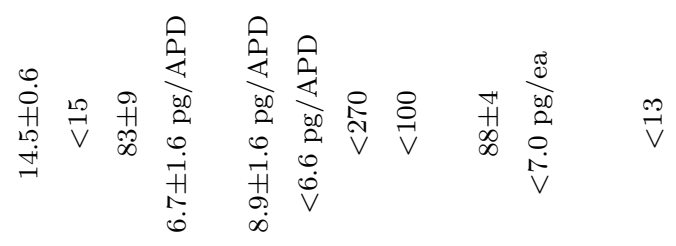

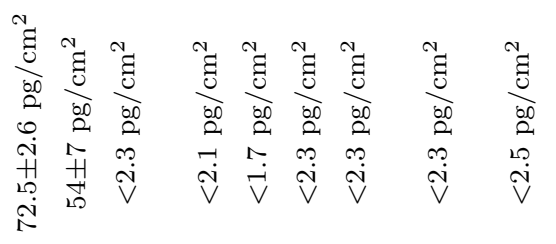

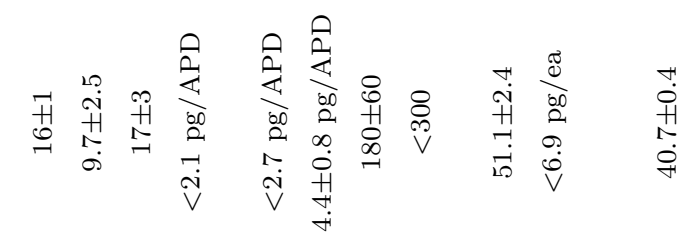

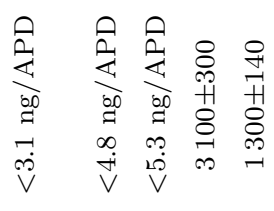

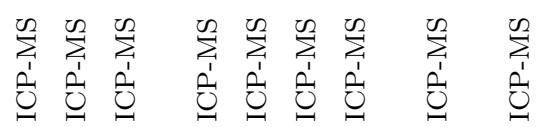

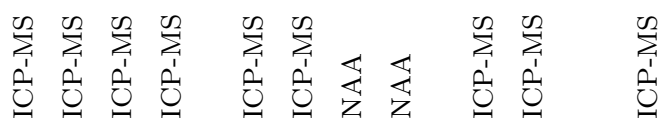
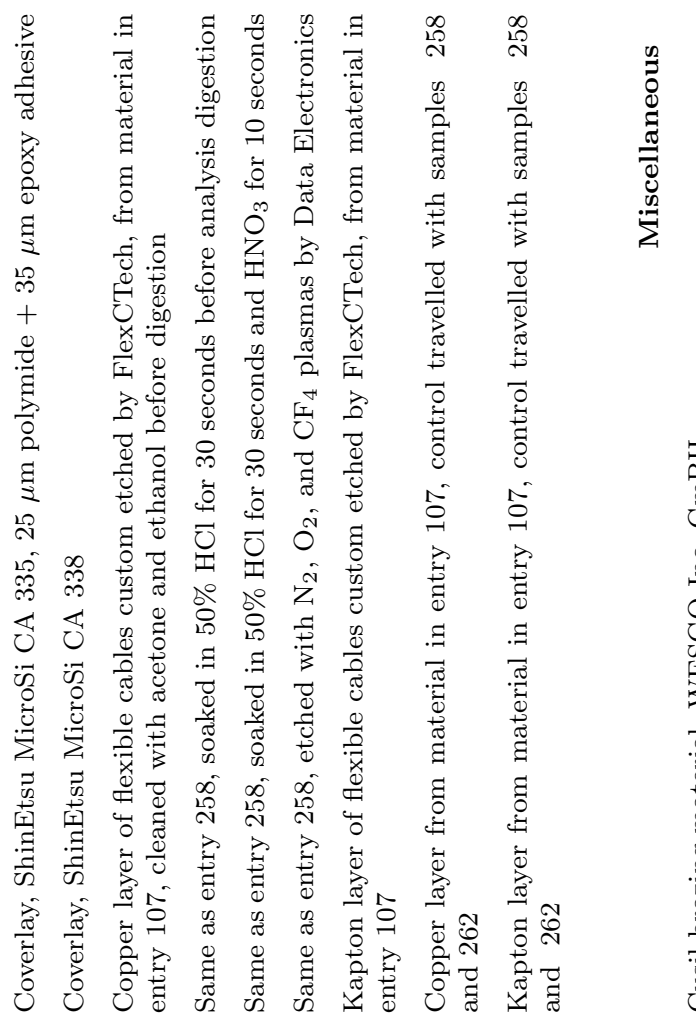

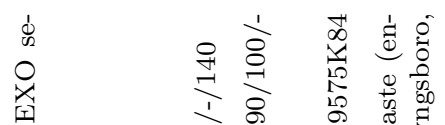

营

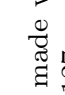

药

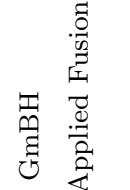

用

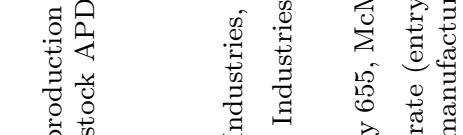

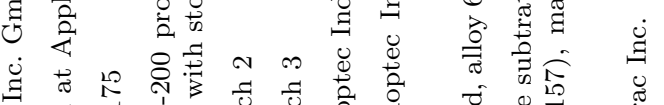

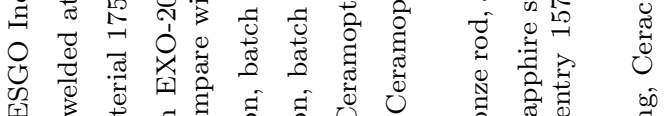

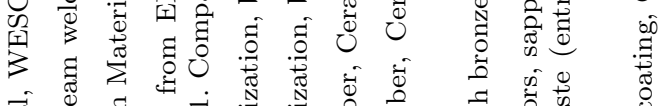

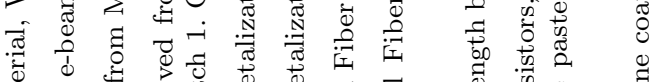

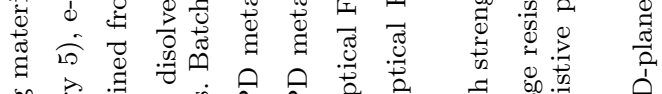

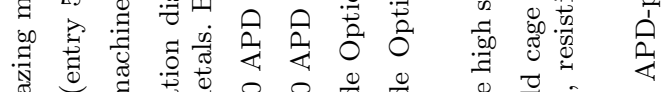

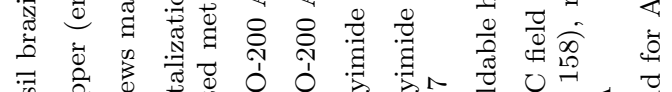

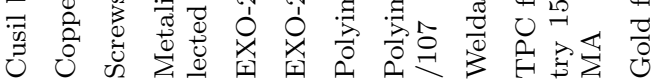

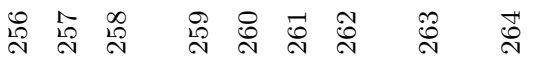

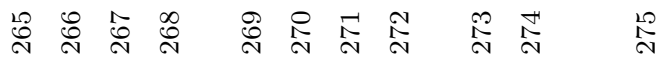




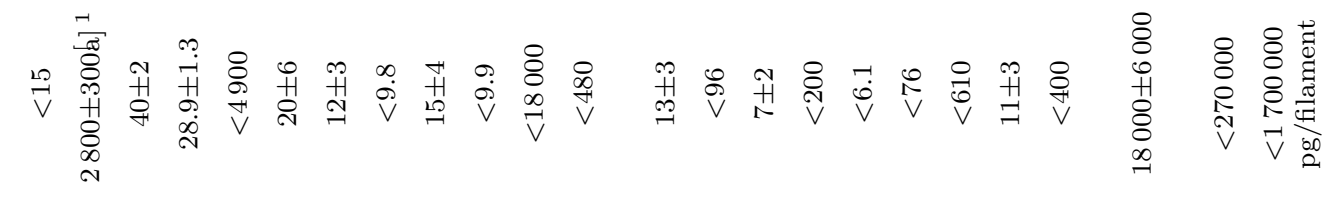

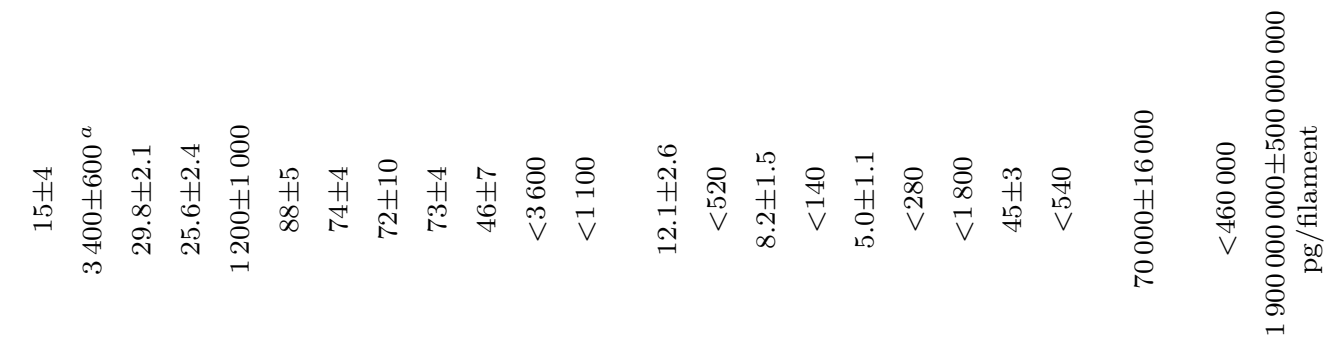

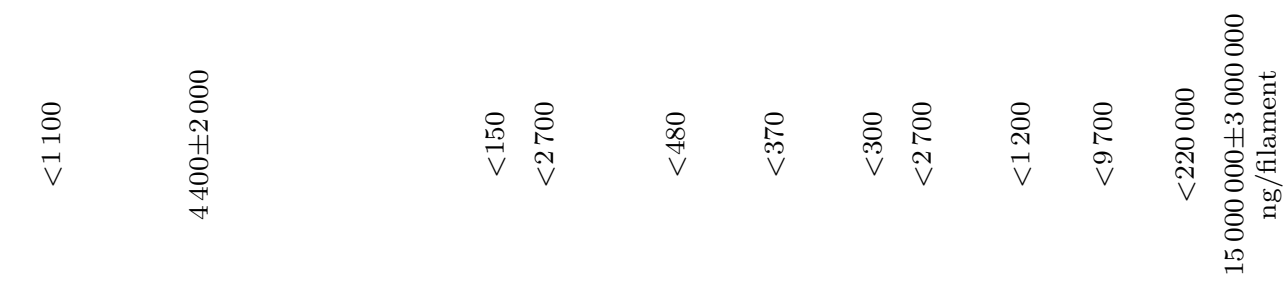

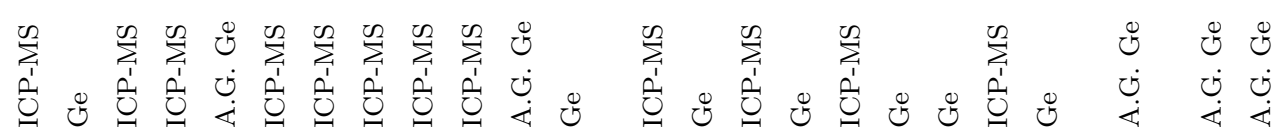

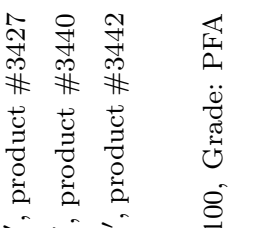

星星教高

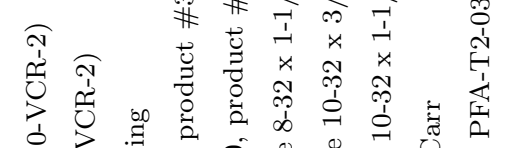

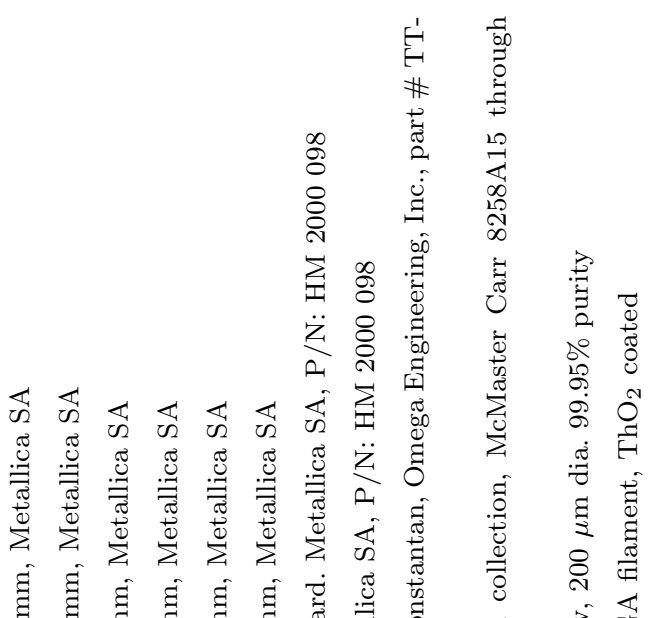

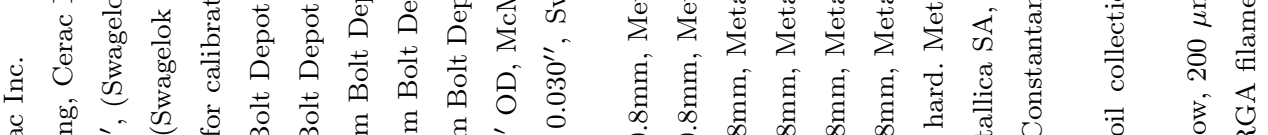

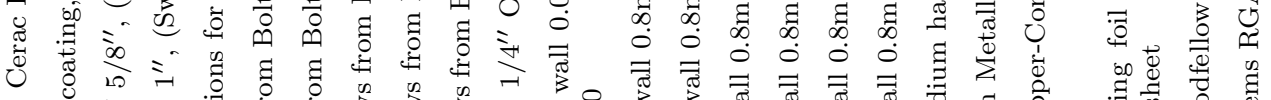

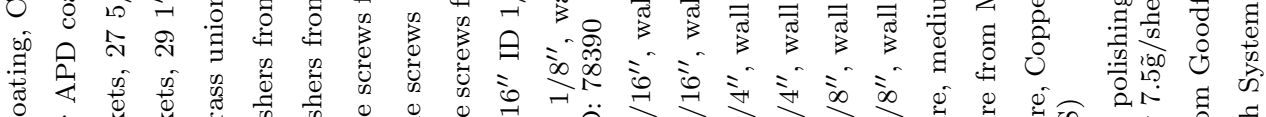

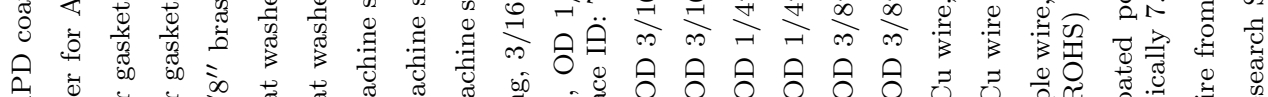

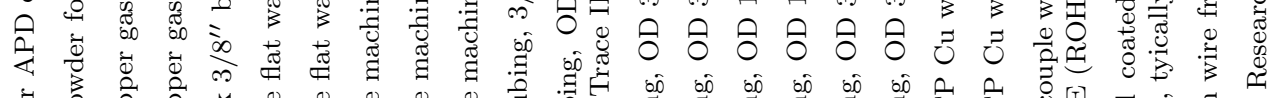

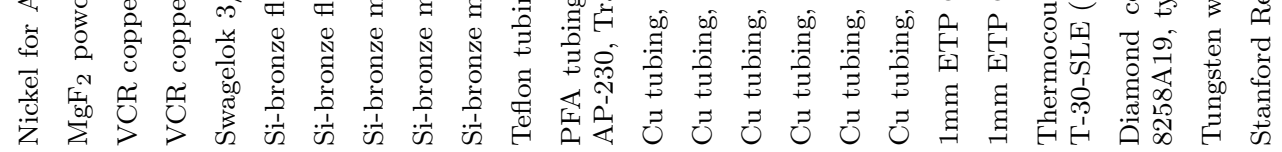

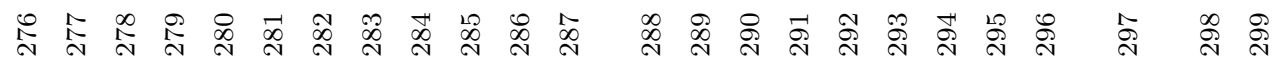




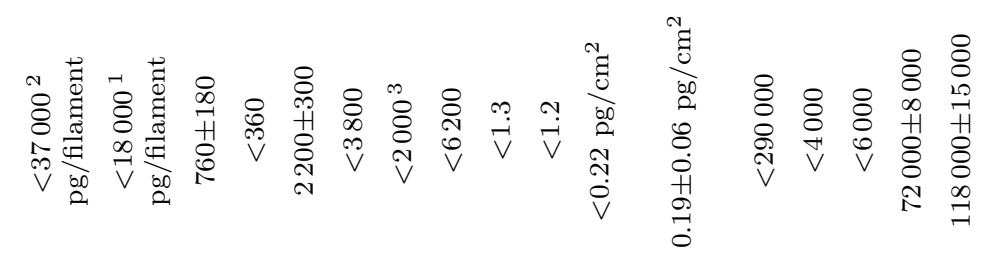

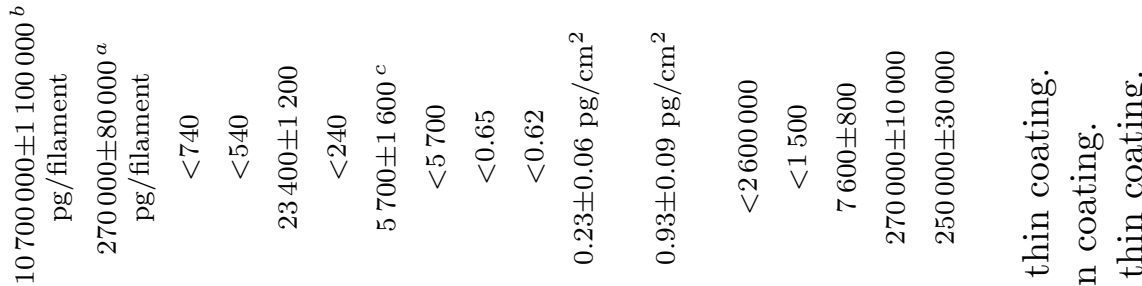

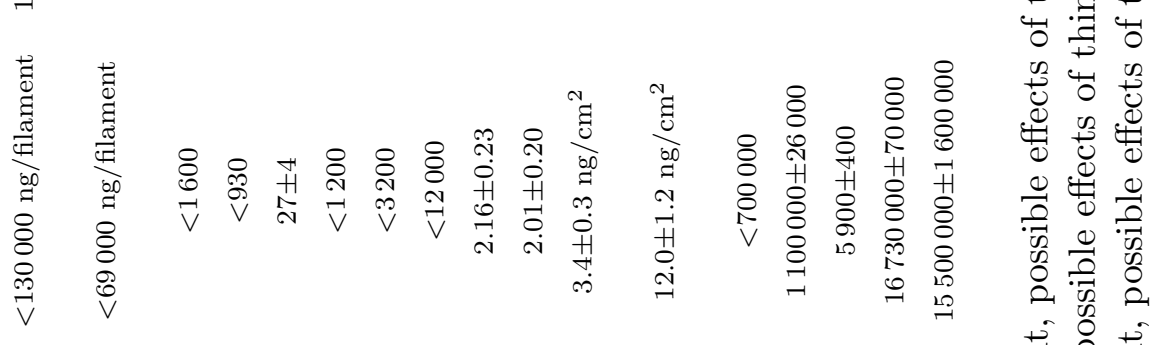

Е

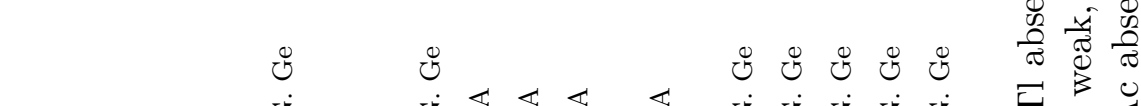

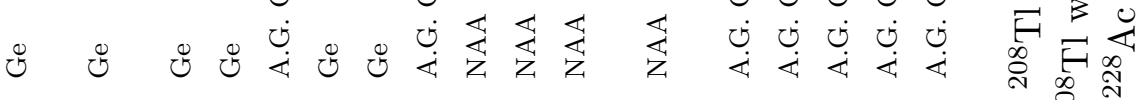

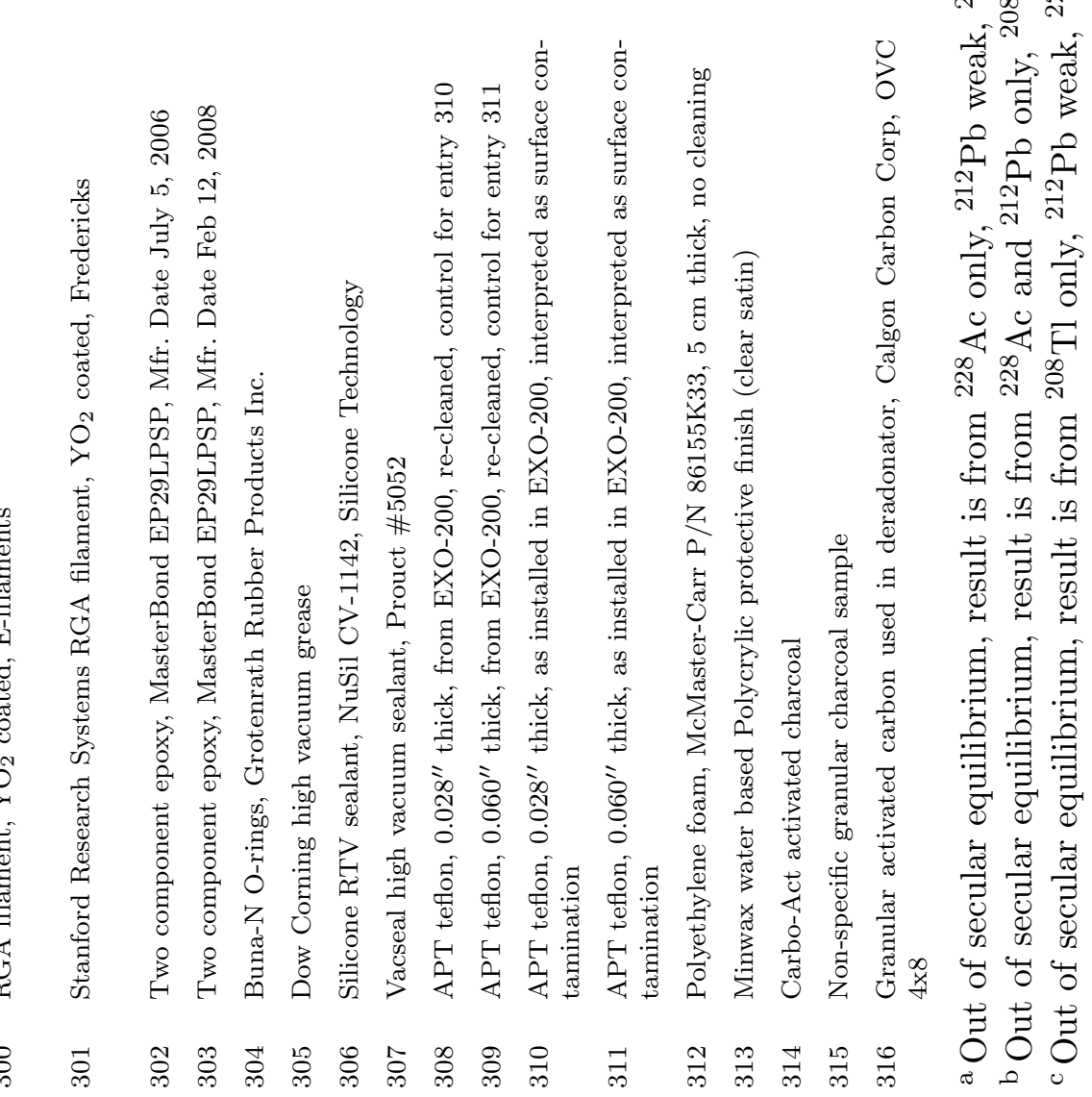




\section{Appendix A Reactor neutrons}

At nuclear reactors the neutron flux shows a broad distribution in Energy, $E$. It is composed of three components: thermal $\Phi_{t h}(E, T)$, epi-thermal $\Phi_{e t}(E)$ and fast fission $\Phi_{f}(E)$ neutrons. The total flux is modeled as the sum of these components: $\Phi(E, T)=\Phi_{t h}(E, T)+\Phi_{e t}(E)+\Phi_{f}(E)$. For a detailed discussion of the modeling of the first two flux components see Ref. [23].

At low neutron energy the flux is described by the Maxwell-Boltzmann distribution, $\Phi_{t h}(E, T)$ :

$$
\Phi_{t h}(E, T) d E=\sqrt{\frac{8}{\pi \cdot m}} \cdot \frac{n}{\left(k_{B} \cdot T\right)^{3 / 2}} \cdot E \cdot e^{-\frac{E}{k_{B} \cdot T}} d E,
$$

where $m$ is the neutron mass, $n$ is the integral neutron density, $k_{B}$ is Boltzmann constant, and $T$ denotes the neutron temperature, assumed to be equal to the temperature of the water moderator.

Here the neutron flux is taken to be proportional to $v_{n} \cdot n_{t h}\left(v_{n}, T\right)$, with $v_{n}$ denoting the neutron velocity and $n_{t h}\left(v_{n}, T\right)$, the Maxwell velocity distribution of the neutron density. The total thermal neutron flux, $\Phi_{t h}$, is defined as: $\Phi_{t h} \equiv \int_{0}^{\infty} \Phi_{t h}(E, T) d E=\left\langle v_{n}\right\rangle \cdot n$, with $\left\langle v_{n}\right\rangle=\sqrt{\frac{8 \cdot k_{B} \cdot T}{\pi \cdot m}}$ denoting the average neutron velocity, evaluated over the velocity distribution.

Ref. [23] states that for neutron energies larger than about $5 \cdot k_{B} \cdot T$ the epithermal spectrum "abruptly" turns on. The epi-thermal flux, $\Phi_{e t}(E)$, is known to be inversely proportional to the neutron kinetic energy: $\Phi_{e t}(E) d E \propto \frac{d E}{E}$. When defining the total epi-thermal neutron flux $\Phi_{e t}$ such that:

$$
\Phi_{e t}=\int_{E_{l}^{e t}}^{E_{u}^{e t}} \Phi_{e t}(E) d E
$$

one obtains the energy dependent epi-thermal neutron flux as:

$$
\Phi_{e t}(E) d E=\frac{\Phi_{e t}}{\ln \left(\frac{E_{e}^{e t}}{E_{l}^{e t}}\right)} \cdot \frac{d E}{E}
$$

To arrive at a finite integrated flux, the epi-thermal flux component can only be defined over integration limits.

At $\mathrm{MeV}$-energies the reactor neutron spectrum is dominated by ${ }^{235} \mathrm{U}$ fast fission neutrons. Because neutron capture cross sections are small at these high energies, including the fast fission flux component typically has a negligible effect on the analysis of NAA data. However, threshold reactions - for example of $(n, p)$ type — on the sample matrix itself can create large sample 
related backgrounds that limit the analysis sensitivity. Inclusion of this flux component is thus useful for the purpose of background estimation. Following Ref. [24], the energy distribution of ${ }^{235} \mathrm{U}$ fast fission neutrons, $\Phi_{f}(E)$, can be modeled by the Watt spectrum:

$$
\Phi_{f}(E) d E=\Phi_{f} \cdot\left[\sqrt{\frac{4}{\pi \cdot a^{3} \cdot b}} \cdot e^{-\frac{a \cdot b}{4}}\right] \cdot e^{-\frac{E}{a}} \cdot \sinh (\sqrt{b \cdot E}) d E
$$

with $a=0.988 \mathrm{MeV}$, and $b=2.249 \mathrm{MeV}^{-1}$. For an upper cut-off energy $\left(E_{u}^{f}\right)$ of $10 \mathrm{MeV}$ or larger (see Ref. [25] for a complete analytic expression for the spectral normalization, the term in square brackets in Equation 5), $\Phi_{f}$ is the integrated fast neutron flux defined as: $\Phi_{f}=\int_{0}^{E_{u}^{f}} \Phi_{f}(E) d E$. At that chosen upper cut-off energy this approximate spectral normalization differs from the complete one by about $0.1 \%$.

\section{A.1 Average cross sections}

The use of cross sections, averaged over the energy distribution of the three flux components discussed before, converts Equation 1 into an algebraic equation. The JENDL-4.0 database [24] conveniently provides such average cross sections, greatly simplifying the interpretation of NAA data. In the following we discuss how to use these tabulated cross sections.

In JENDL-4.0 the thermal average cross section is defined as:

$$
\sigma_{t h}\left(T_{0}\right) \equiv \frac{2}{\sqrt{\pi}} \cdot \frac{\int_{E_{l}^{t h}}^{E^{t h}} \sigma(E) \cdot \Phi_{t h}\left(E, T_{0}\right) d E}{\int_{E_{l}^{t h}}^{E_{t h}^{t h}} \Phi_{t h}\left(E, T_{0}\right) d E} \approx \frac{2}{\sqrt{\pi}} \cdot \frac{Y_{t h}}{\Phi_{t h}},
$$

where $T_{0}=300 \mathrm{~K}$ is the reference temperature. The lower and upper integration limits are $E_{l}^{t h}=10^{-5} \mathrm{eV}$ and $E_{u}^{t h}=10 \mathrm{eV}$, respectively. $Y_{t h}$ is the thermal neutron induced reaction yield per atom. For the chosen integration limits the integral in the denominator of Equation 6 differs from $\Phi_{t h}$ by order $10^{-6}$. The factor of $2 / \sqrt{\pi}$ equals the ratio of the average over the most likely thermal neutron velocity for a Maxwell-distribution. It normalizes the total thermal neutron flux to the traditional notation which equates the integral flux to the volume density times the most likely neutron velocity.

To model the capture of epi-thermal neutrons JENDL-4.0 provides the socalled resonance integral (RI). The RI is defined as:

$$
\sigma_{R I} \equiv \int_{E_{l}^{e t}}^{E_{u}^{e t}} \sigma(E) \frac{d E}{E}
$$


with $E_{l}^{e t}=0.5 \mathrm{eV}$ and $E_{u}^{e t}=10 \mathrm{MeV}$. Because of the missing spectral-flux normalization, the tabulated RI is not an average cross section in a mathematical sense. Substituting Equation 4 into Equation 7 relates the RI to the epi-thermal neutron capture yield $Y_{\text {et }}$ :

$$
\sigma_{R I}=\ln \left(\frac{E_{u}^{e t}}{E_{l}^{e t}}\right) \cdot \frac{\int_{E_{l}^{e t}}^{E_{e}^{e t}} \sigma(E) \cdot \Phi_{e t}(E) d E}{\Phi_{e t}} \approx 16.81 \cdot \frac{Y_{e t}}{\Phi_{e t}}
$$

From Equation 8 one can see that a numerical factor is needed to relate the epi-thermal neutron capture yield to the resonance integral and epi-thermal flux integral.

The average fast fission capture cross section, $\sigma_{f}$ is defined as a proper mathematical average and relates directly to the capture yield $Y_{f}$ per target atom:

$$
\sigma_{f} \equiv \frac{\int_{E_{l}^{f}}^{E_{u}^{f}} \sigma(E) \cdot \Phi_{f}(E) d E}{\int_{E_{l}^{f}}^{E_{u}^{f}} \Phi_{f}(E) d E} \approx \frac{Y_{f}}{\Phi_{f}}
$$

with $E_{u}^{f}=10^{-5} \mathrm{eV}$ and $E_{u}^{f}=20 \mathrm{MeV}$.

Using Equations 6, 8, and 9, Equation 1 can be re-written using the average cross sections and integral neutron fluxes:

$$
R_{D}(t)=N_{T} \cdot\left(1-e^{-t_{i} / \tau_{D}}\right) \cdot e^{-t / \tau_{D}} \cdot\left[\sigma_{t h}(T) \cdot \Phi_{t h}+\frac{\sigma_{R I}}{16.81} \cdot \Phi_{e t}+\sigma_{f} \cdot \Phi_{f}\right]
$$

$\Phi_{R I}=\frac{\Phi_{e t}}{16.81}$ is called the resonance integral flux. Numerical calculation of average cross sections done in the course of this work and using energy dependent neutron capture cross sections for ${ }^{23} \mathrm{Na},{ }^{41} \mathrm{~K},{ }^{50} \mathrm{Cr},{ }^{58} \mathrm{Fe},{ }^{59} \mathrm{Co},{ }^{64} \mathrm{Zn}$, ${ }^{197} \mathrm{Au},{ }^{232} \mathrm{Th}$ and ${ }^{238} \mathrm{U}$, obtained online from Brookhaven National Laboratory typically agreed to within a few percent with the JENDL-4.0 tabulated values.

The water moderator at MITR II has a water temperature of about $T=340 \mathrm{~K}$, $11 \%$ higher than the JENDL-4.0 reference temperature. For the nuclides listed before this results in a $6 \%$ smaller thermal neutron capture rate compared to the JENDL-4.0 reference temperature, calculated using Equation 6. The change corresponds to a factor of $\sqrt{\frac{T_{0}}{T}}$, as expected for thermal neutron capture cross sections which are proportional to $1 / v_{n}$. The data is corrected for this temperature dependence.

\section{A.2 The reactor neutron flux}

Integral neutron fluxes are needed in Equation 10 to determine $N_{T}$. The neutron flux at MITR's 2PH1 irradiation facility has been determined by ac- 


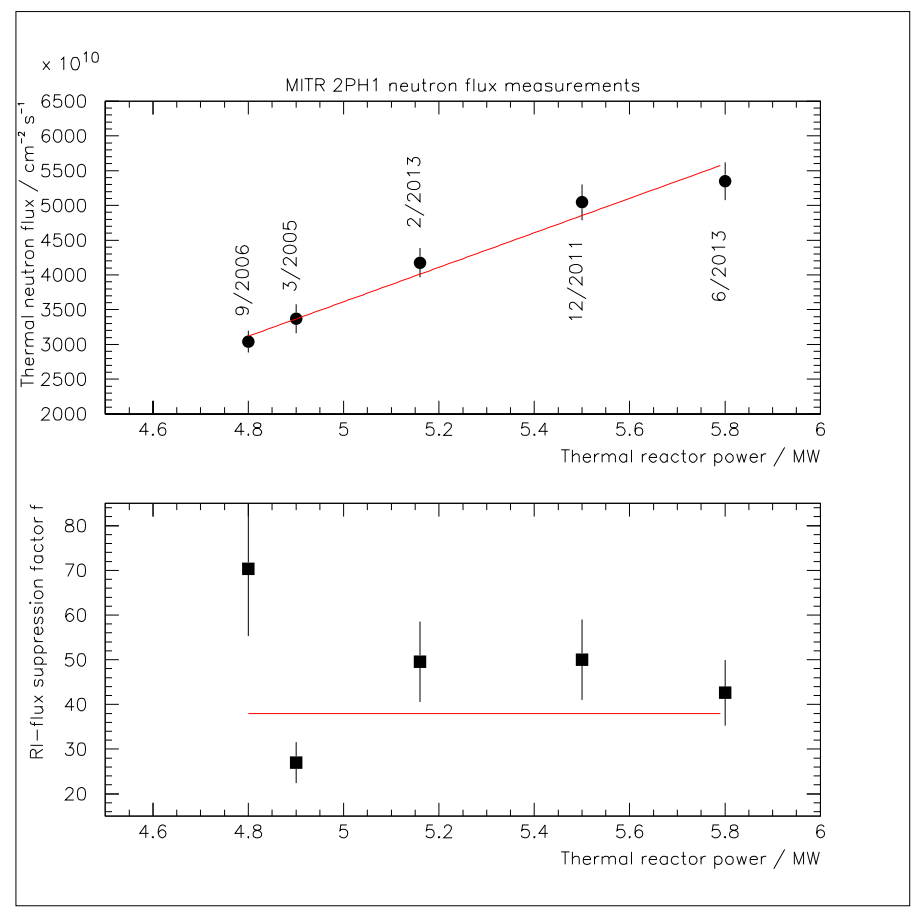

Fig. 4. Thermal neutron flux (top) and ratio of RI-flux over thermal flux (bottom) as determined from fly ash activations as a function of thermal reactor power.

tivating NIST-certified fly ash (SRM 1633b), containing known amounts of activatable nuclides. $\mathrm{Na}, \mathrm{K}, \mathrm{Ti}, \mathrm{Cr}, \mathrm{Fe}$, Th and $\mathrm{U}$ are used in a combined fit. The activities of all isotopes, $j$, are determined by Ge detector-based gammaray spectroscopy. Multiple gamma lines are used, whenever possible, in a time and energy differential fit, described in Sec. 5.2. The parameterized values $A_{j}^{P}\left(t_{d}\right)$ of the activities at the reference time $t_{d}$ after end of irradiation are:

$$
A_{j}^{P}\left(t_{d}\right)=N_{T_{j}} \cdot\left(1-e^{-t_{i} / \tau_{D_{j}}}\right) \cdot e^{-t_{d} / \tau_{D_{j}}} \cdot\left[\sigma_{t h}(T) \cdot \Phi_{t h}+\sigma_{R I} \cdot \Phi_{R I}+\sigma_{f} \cdot \Phi_{f}\right],
$$

and the integral neutron fluxes are determined by minimizing:

$$
\chi^{2}=\sum_{j=1}^{7} \frac{\left[A_{j}^{O}\left(t_{d}\right)-A_{j}^{P}\left(t_{d}, t_{i}, \Phi_{t h}, \Phi_{R I}, \Phi_{f}\right)\right]^{2}}{\sigma_{j}^{2}}
$$

with respect to the three neutron fluxes, for the observed activities $A_{j}^{O}$. The 
dependence of $A_{j}^{P}\left(t_{d}\right)$ on the irradiation parameters has now been made explicit, and the fluxes $\Phi_{t h}, \Phi_{R I}$ and $\Phi_{f}$ are the free floating fit parameters. The number of target atoms and their errors, $N_{T_{j}}$ and $\sigma_{N_{T_{j}}}$ are derived from the NIST certified elemental masses of the fly ash components and their errors. Molar masses and isotope abundances are taken from [26], the nuclide life times $\tau_{D_{j}}$ from [27]. These parameters have negligible uncertainties; their errors are not propagated. Practically the determination of $\Phi_{R I}$ is driven by

${ }^{232} \mathrm{Th}$ and ${ }^{238} \mathrm{U}$ due to their large resonance integrals. ${ }^{48} \mathrm{Ti}(n, p){ }^{48} \mathrm{Sc}$ determines $\Phi_{f}$. Due to this decoupling, the one-simga errors on the fluxes are determined as one-parameter errors, corresponding to $\Delta \chi^{2}=1$. The variances $\sigma_{j}^{2}$ contain the statistical counting error, a $10 \%$ systematic added in quadrature to activities (meant to measure variability of the counting solid angle), and the error of the masses of the components of the fly ash, as certified by NIST.

The thermal and epi-thermal neutron fluxes at MITR have been determined multiple times using the fly ash method. Figure 4 shows $\Phi_{\text {th }}$ (top) and $f=$ $\Phi_{R I} / \Phi_{t h}$ (bottom) as a function of thermal reactor power. These data were collected between 2005 and 2013. The data shown in the top panel of Figure 4 indicates a linear correlation between thermal neutron flux and reactor power, as long as the power is above a $4.8 \mathrm{MW}$ threshold. Data collected at lower reactor power does not follow this systematic. However, for the data collected here this is irrelevant as these activations had their own flux calibrations and did not rely on the flux-power fit. A linear fit to the $2 \mathrm{PH} 1$ data above $4.8 \mathrm{MW}$ yields a slope of $(2.48 \pm 0.27) \cdot 10^{13} \mathrm{~cm}^{-2} \mathrm{~s}^{-1} \mathrm{MW}^{-1}$, an intercept of $(-8.8 \pm$ 1.4) $\cdot 10^{13} \mathrm{~cm}^{-2} \mathrm{~s}^{-1}$ with an error correlation coefficient of -0.998 . The average RI-flux suppression factor is found to be $38.0 \pm 3.2$.

The fast neutron flux has only been measured twice at 2PH1; a correlation with the reactor power cannot yet be established. For the activation at the highest neutron flux $(6 / 2013)$ we determined: $\Phi_{f}=(3.3 \pm 0.9) \cdot 10^{12} \mathrm{~cm}^{-2} \mathrm{~s}^{-1}$ from fly ash data. In addition a sample of TiN was activated, constraining $\Phi_{f}$ via the reactions ${ }^{46} \mathrm{Ti}(n, p){ }^{46} \mathrm{Sc}$ and ${ }^{48} \mathrm{Ti}(n, p){ }^{48} \mathrm{Sc}$. This independent measurement yielded $\Phi_{f}=(2.4 \pm 0.2) \cdot 10^{12} \mathrm{~cm}^{-2} \mathrm{~s}^{-1}$, averaged over both Sc-activities, and is in agreement with the fly ash data.

\section{Acknowledgements}

EXO-200 is supported by DOE and NSF in the United States, NSERC in Canada, SNF in Switzerland, IBS in Korea, RFBR in Russia, DFG Cluster of Excellence "Universe" in Germany, and CAS and ISTCP in China. 


\section{References}

[1] M. Danilov, et al., Phys. Lett. B 480 (2000) 12; M. Breidenbach, et al., Letter of intent to SLAC EPAC, LOI-2001.1, August 2001, unpublished.

[2] D. Leonard, et al., Nucl. Inst. Meth. A 591 (2008) 490.

[3] C. Arpesella, et al., Astro. Part. Phys. 18 (2002) 1.

[4] M. Laubenstein, et al., Appl. Radiat. Isot. 61 (2004) 167.

[5] D. Budjas, et al., Appl. Radiat. Isot. 67 (2009) 775.

[6] E. Aprile, et al., Astropart. Phys. 35 (2011) 43.

[7] E. Armengaud, et al., Astropart. Phys. 47 (2013) 1.

[8] V. Álvarez, et al., J. Inst. 8 (2013) T01002.

[9] N. Abgrall, et al., Nucl. Inst. Meth. A 828 (2016) 22.

[10] X. Wang, et al., J. Instrum. 11 (2016) T12002.

[11] M. Auger, et al., Rev. Sci. Instrum. 7 (2011) 05010.

[12] N.Ackerman, et al., Phys. Rev. Lett. 107 (2011) 212501.

[13] M. Auger, et al., Phys. Rev. Lett. 109 (2012) 032505.

[14] J. B. Albert, et al., Nature 510 (2014) 229.

[15] J. B. Albert, et al., Phys. Rev. C 89 (2014) 015502.

[16] M. Redshaw, E. Wingfield, J. McDaniel, E. Myers, Phys. Rev. Lett. 98 (2007) 053003 .

[17] J. B. Albert, et al., Phys. Rev. C 92 (2014) 015503.

[18] Y. Gonin, J. Busto, J.-L. Vuilleumier, Rev. Sci. Instrum. 74 (2003) 4663.

[19] D. Reusser, et al., Phsy. Rev. D 45 (1992) 2548.

[20] Nuclear Data Sheets 121 (2014) 561.

[21] P. Grinberg, S. Willie, R. E. Sturgeon, Analytical Chemistry 77 (2005) 2432.

[22] B. LaFerriere, T. Maiti, I. Arnquist, E. Hoppe, Nucl. Inst. Meth. A 775 (2015) 93.

[23] C. Wescott, Nucl. Eng. 2 (1955) 59.

[24] K. Shibata, et al., J. Nucl. Sci. Technol 48 (2011) 1, http://www.ndc.jaea.go.jp/jendl/j40/j40.html.

[25] A. Trkov, M. Herman, D. Brown, Report bnl-90365-2009 rev. 2, Tech. rep., National Nuclear Data Center (2009).

[26] R. B. Firestone, S. Chu, Table of isotopes cd-rom (1998).

[27] http://www.nndc.bnl.gov/chart/. 